\title{
A molecular code for endosomal recycling of phosphorylated cargos by the SNX27-retromer complex
}

Thomas Clairfeuille ${ }^{1,2}$, Caroline Mas ${ }^{1,3}$, Audrey S.M. Chan ${ }^{4}$, Zhe Yang ${ }^{1}$, Maria Tello-Lafoz ${ }^{5}$, Mintu Chandra ${ }^{1}$, Jocelyn Widagdo ${ }^{6}$, Markus C. Kerr ${ }^{1}$, Blessy Paul ${ }^{1}$, Isabel Mérida ${ }^{5}$, Rohan D. Teasdale ${ }^{1}$, Nathan J. Pavlos ${ }^{4}$, Victor Anggono ${ }^{6}$, and Brett M. Collins ${ }^{1^{*}}$

1. Institute for Molecular Bioscience, The University of Queensland, St. Lucia, Queensland, 4072, Australia.

2. Present address: Genentech Inc., 1 DNA way, 94080, South San Francisco, California, USA.

3. Present address: Integrated Structural Biology Grenoble (ISBG), UMS 3518 (CNRS-CEA-UJF-EMBL), 71 avenue des Martyrs, 38042 Grenoble Cedex 9, France.

4. Cellular Orthopaedic Laboratory, School of Surgery, The University of Western Australia, Nedlands, WA, 6009, Australia.

5. Lipid Signalling Laboratory, Centro Nacional de Biotecnología (CNB)/CSIC, E-28409, Madrid, Spain.

6. Clem Jones Centre for Ageing Dementia Research, Queensland Brain Institute, The University of Queensland, Brisbane, QLD 4072, Australia.

* Correspondence should be addressed to BMC (e-mail: b.collins@imb.uq.edu.au).

Running Title: SNX27-retromer binds phosphorylated cargo

Keywords: endosome, PDZ domain, retromer, SNX27, solute carrier, sorting nexin, phosphorylation

Competing interests statement. The authors declare that they have no competing financial interests. 


\section{SUMMARY}

Recycling of internalised receptors from endosomal compartments is essential for their cell surface homeostasis. Sorting nexin 27 (SNX27) cooperates with the retromer complex in the recycling of proteins containing type I PSD95/DIg/ZO1 (PDZ) binding motifs. Here we define specific acidic amino acid sequences upstream of the PDZ motif required for high affinity engagement of the SNX27 PDZ domain. However, a subset of SNX27 ligands such as the $\beta_{2^{-}}$ adrenergic and N-methyl-D-aspartate (NMDA) receptors lack these sequence determinants. Instead we identify conserved sites of phosphorylation that substitute for acidic residues to dramatically enhance SNX27 interactions. This newly identified mechanism points to a likely regulatory switch for PDZ interaction and protein transport by SNX27-retromer. Defining this SNX27-binding code has allowed us to classify more than four hundred potential SNX27 ligands with broad functional implications in signal transduction, neuronal plasticity and metabolite transport. 


\section{INTRODUCTION}

Endocytic recycling of internalised transmembrane proteins is essential for their homeostasis, but the mechanisms that regulate sequence-dependent recycling are still poorly understood. The retromer trafficking complex recycles cargos containing PSD95/Dlg/ZO1 (PDZ) domain binding motifs via the adaptor protein sorting nexin 27 (SNX27). PDZ domains are one of the most common protein:protein interaction scaffolds found in proteins controlling cell trafficking and signalling networks ${ }^{1,2}$, and play a significant role in clustering of neuronal receptors within the post-synaptic density (PSD) ${ }^{3}$. The canonical mechanism of PDZ domain interaction involves a conserved cavity that recognizes different classes of PDZ binding motifs (PDZbms) found at the C-terminus of ligand proteins. Type 1 motifs have the sequence $[S / T]-X-\Phi(\Phi=$ any hydrophobic residue), often found within the cytosolic tail of transmembrane proteins ${ }^{4}$. While the C-terminal triplet is essential for binding, specificity is often enhanced by upstream sequences ${ }^{5,6}$.

Non-canonical PDZ domain binding is less common, and involves extensions to the core PDZ fold that then promote interactions with specific protein partners ${ }^{7}$. SNX27 uses both canonical and noncanonical PDZ interfaces to simultaneously engage Type 1 PDZ ligands and the endosomal trafficking hub complex retromer (a heterotrimer of VPS35, VPS26, and VPS29) via the VPS26 subunit ${ }^{8}$ (Fig. 1A). It thus acts as a cargo adaptor for retromer-mediated transport from endosomes to the cell surface ${ }^{8-11}$. Cargos include G-protein coupled receptors (GPCRs) such as the $\beta 2$-adrenergic receptor $\left(\beta_{2} A R\right){ }^{10,12}$ and parathyroid hormone receptor (PTHR) ${ }^{13}$, ion channels ${ }^{14,15}$, and proteins involved in neuronal plasticity including $\alpha$-amino-3-hydroxy-5-methyl-4-isoxazolepropionic acid (AMPARs) ${ }^{16,17}$, N-methyl-Daspartate (NMDARs) ${ }^{18}$ and 5-hydroxytryptamine 4 a receptors $\left(5-\mathrm{HT}_{4(\mathrm{a})} \mathrm{R}\right){ }^{19}$ among others ${ }^{9,20}$.

SNX27 and retromer are expressed in multiple tissues, but play particularly prominent roles in neurons. Altered expression and mutations are linked to Alzheimer's Disease, Parkinson's Disease, Down Syndrome and infantile epilepsy ${ }^{18,21-28}$, and lowered expression of SNX27 or retromer reduces surface levels of $\beta 2 A R s$, NMDARs and AMPARs with correlated synaptic defects ${ }^{12,17,18}$. It has also been found that SNX27 and retromer play a role in bone morphogenesis through trafficking of the PTHR in osteoblasts ${ }^{13}$. Generally however, it remains poorly understood as to how SNX27-retromer efficiently recruits diverse endosomal cargos (and accessory proteins), which are often transported in response to activation by different cell stimuli.

Here we define sequence requirements for PDZbm binding to the SNX27-retromer complex and show that ligands fall into two distinct categories of high and low affinity binders based on the positions of acidic side chains upstream of the C-terminal PDZbm. High affinity-cargos require acidic residues located three and five residues upstream of the C-terminus that are able to clamp a conserved arginine on the SNX27 surface. Most intriguing is that many SNX27 ligands, including the $\beta 2 A R$ and NMDARs, lack these upstream acidic side-chains, but instead possess conserved sites of serine and threonine phosphorylation. Data both in vitro and in cells, together with the crystal structures of SNX27 bound to different phosphopeptides, shows that phosphorylation of these residues mimics acidic side-chains 
required for high affinity binding, and points to a novel layer of post-translational regulation in endosomal recycling. In silico analyses of the human genome provides a comprehensive inventory of probable SNX27-retromer interactors, and suggests there are hundreds of potential proteins recycled by SNX27retromer either constitutively or following phosphorylation. 


\section{RESULTS}

\section{Specific sequences in PDZbms control association with SNX27}

A common feature seen in previous structures of the PDZbms of PTHR and Kir3.3 bound to SNX27 is the presence of what we refer to as an 'electrostatic plug', where two glutamate side chains at the -3 and -5 positions of the peptides embrace the conserved SNX27 residue Arg58 ${ }^{13,14}$ (Fig. 1B). In addition SNX27 possesses an extended surface of positive electrostatic potential adjacent to the -5 and -6 peptide side-chains. Our prediction was that PDZbm sequences containing the acidic pair of side chains at -3 and -5 would bind more strongly than those without, and that acidic side-chains at -6 might enhance the binding affinity through delocalised electrostatic association.

To define the relative contribution of each acidic residue in binding to SNX27, we used the PTHR PDZbm as a model peptide ${ }^{13}$ and measured the affinities of a series of alanine mutations (Fig. 1C; Table S1). This showed: (i) altering the $\mathrm{Glu}^{-6}$ residue (PTHR-A1) on its own does not significantly reduce SNX27 binding, (ii) Glu ${ }^{-5}$ substitution (PTHR-A2), predicted to destabilize the electrostatic plug, leads to a modest decrease in affinity from $6 \mu \mathrm{M}$ to $15 \mu \mathrm{M}$, and (iii) the elimination of both $\mathrm{Glu}^{-5}$ and $\mathrm{Glu}^{-6}$ (PTHRA3) reduces the binding affinity even further. Hence it appears that while $\mathrm{Glu}^{-5}$ is most important, a combined negative potential at both -5 and -6 can further enhance interaction. Since the PDZ binding pocket is surrounded by positively charged side-chains near the $\mathrm{N}$-terminus of the bound peptide (see Fig. 1B), and the acidic residue at -6 is not ordered in the PTHR complex (or DGK $\zeta$, as shown below), we propose that a localised electrostatic complementarity between $\mathrm{N}$-terminal PDZbm acidic side-chains and the basic surface of SNX27 is not strictly required, but can enhance peptide interactions. We previously showed that $\mathrm{Glu}^{-3}$ to $\mathrm{Ala}^{-3}$ mutation (PTHR-A5/A6) or a C-terminal Ala addition (PTHR-A4) prevented association with SNX27 ${ }^{13}$ (shown in Fig. 1C for comparison), indicating that Glu ${ }^{-3}$ and its contacts with Arg58 and Asn56 are essential for complex formation as is the register of the C-terminal PDZbm (Fig. 1C).

We next tested the importance of specific PTHR sequences for SNX27-retromer association in cells. HEK293 cells stably expressing untagged PTHR were stimulated with fluorescent tetramethylrhodamine (TMR) labelled $\mathrm{PTH}(1-34)$ analog $\left(\mathrm{PTH}^{\mathrm{TMR}}\right)$ to induce activation and receptor internalisation. Endocytosed PTH ${ }^{\mathrm{TMR}}$-bound PTHR showed significant association with SNX27 at endosomes after several minutes as observed previously ${ }^{13}$ (Fig. 1D). However, when the C-terminal sequences of PTHR were mutated at the $-3 /-5$ positions or to remove the PDZbm altogether ${ }^{13}$, we found that PTHR was readily internalised following stimulation and localised to endocytic compartments, however, both showed a similar defect in colocalisation with SNX27 as determined by Pearson's correlation co-efficient $(R r)$ ((Fig. 1E).

\section{Electrostatic interactions promote high affinity PDZbm association with SNX27}

We next tested the binding of three other ligands known to be associated with the SNX27-retromer pathway: (i) $\beta_{2} A R{ }^{11}$, (ii) diacylglycerol kinase $\zeta$ (DGK $\zeta$ ), a soluble SNX27-binding enzyme ${ }^{29,30}$, and (iii) 
the scaffolding protein Kinase D-interacting substrate of $220 \mathrm{kDa}$ (KIDINS220) ${ }^{9}$. The corresponding PDZbm peptides showed large differences in their ability to bind SNX27 PDZ. $\beta_{2} A R$ association was barely detectable $\left(K_{d}>200 \mu \mathrm{M}\right)$, whereas both KIDINS220 and DGK $\zeta$ interacted strongly $\left(K_{d} \sim 6.5 \mu \mathrm{M}\right.$ and $2 \mu \mathrm{M}$ respectively) (Fig. 2A, 2B; Table S1). Mutation of the SNX27 peptide-binding pocket (H114A) blocks all interactions, confirming the specificity. The magnitude of the enthalpic energy release $(\Delta H)$ on DGK $\zeta$ or KIDINS220 binding is many times bigger that of $\beta_{2} A R$, suggesting electrostatic bonds make a larger contribution to their interaction. Similar results are seen for the $\mathrm{PH}$ Domain And Leucine Rich Repeat Protein Phosphatase 1 (PHLPP1) and PHLPP2 sequences, that our bioinformatics studies predict should bind (see below), and were observed in proteomics ${ }^{31}$ (Fig. 2C; Table S1). VPS26 allosterically improves binding to these PDZbms as shown previously for GLUT1, Kir3.3 ${ }^{8}$ and PTHR ${ }^{13}$, and this is markedly pronounced for $\beta_{2} A R$.

To confirm that upstream acidic residues promote high affinity PDZbm interaction with SNX27 we mutated the $-5 /-6$ residues in $\beta_{2} A R$ and DGK $\zeta$ from ST to EE and ED to SS respectively (Fig. 2A). In DGK $\zeta$ this caused a decrease in affinity $\left(K_{d}\right.$ from 2.0 to $\left.5.4 \mu \mathrm{M}\right)$. The reverse substitution of $\mathrm{Glu}^{-5 /-6}$ in $\beta_{2} A R$ dramatically improved binding ( $K_{d}$ from $>200 \mu \mathrm{M}$ to $19 \mu \mathrm{M}$ ) (Fig. 2A). Overall, these results show that acidic residues in $-5 / 6$ positions are not strictly required, but can contribute significantly to the affinity of PDZbm interaction with SNX27.

\section{Structure of the SNX27 PDZ domain bound to DGK $\zeta$ confirms the electrostatic plug binding mechanism}

To investigate PDZbm recognition by SNX27 in more depth, we determined the crystal structure of the SNX27 PDZ domain bound to the PDZbm from DGK $\zeta$ at $1.1 \AA$ resolution (Fig. 3, Table S2). The first $\mathrm{Arg}^{-7}$ residue in the $D G K \zeta$ peptide is not visible in the electron density, and the $\mathrm{Glu}^{-6}$ side chain has relatively poor density and does not interact directly with the PDZ domain, strengthening the idea that residues upstream of the -5 position are not stably associated with SNX27 (Fig. 3A). The three DGK $\zeta$ Cterminal residues engage SNX27 in the canonical orientation where the terminal carboxyl group of $\mathrm{Val}^{0}$ forms critical hydrogen bonds with the backbone of SNX27 Tyr53 and Gly54, while its side chain is embraced by a hydrophobic cavity underneath. Thr ${ }^{-2}$ forms a hydrogen bond with SNX27 His114 (Fig. $3 \mathrm{C}$, right panel), that is essential for complex formation.

Determining SNX27/PDZbm structures at atomic resolution allowed us to compare the detailed molecular aspects of PTHR ${ }^{13}$, Kir3.3 ${ }^{14}$ and DGK $\zeta$ binding (Fig. 3B). All peptides have a Glu ${ }^{-3}$ residue sandwiched between SNX27 Arg58 and Asn56 that also forms a hydrogen bond with Ser82. These strong contacts explain why $\mathrm{Glu}^{-3}$ is the major determinant of selectivity. The other prominent feature of this interaction is how Arg58 establishes contact with both $\mathrm{Glu}^{-3}$ and $\mathrm{Glu} / \mathrm{Asp}^{-5}$ of these peptides forming an electrostatic 'plug'. The salt bridges shared between these three residues lock the peptides in place upstream of the PDZ cavity. Altogether, these combined structures show that the $\mathrm{Glu}^{-3}$ residue is 
essential for binding, while the electrostatic 'plug' involving an acidic side-chain at the PDZbm -5 position provides a mechanism for enhanced selectivity.

To confirm the importance of the electrostatic plug in DGK $\xi$ we tested the ability of DGK mutants to co-immunoprecipitate SNX27 in Jurkat T-cells. While GFP-DGK $\zeta$ precipitated SNX27, constructs modified in their PDZ triplet did not ( $\mathrm{E} T \mathrm{TAV}$, T927A, T927D) (Fig. 3D). Mutating the -3 side chain (E926A or D924A/E926A) abolished the interaction with SNX27, while altering the -5 side-chain on its own (D924A) reduced but did not abolish binding, indicating the -5 acidic side-chain plays an important but auxiliary role to the essential -3 residue. This also correlates with a mild reduction in binding affinity by ITC when altering the -6 and -5 residues (E923S/D924S; ED/SS) (Table S1).

\section{Phosphorylation of PDZbms promotes interaction with SNX27-retromer}

Trafficking is frequently regulated by post-translational modifications including ubiquitination and phosphorylation. While SNX27 regulates PDZ-motif-dependent endosomal recycling of GPCRs such as $\beta 2 A R^{11}$ and $5-\mathrm{HT}_{4(\mathrm{a})} \mathrm{R}{ }^{19}$, as well as AMPARs and NMDARs ${ }^{16-18}$, it is notable that none of these important cargos possess the acidic residues required for forming the stable electrostatic plug with Arg58. However, these proteins do possess sites of potential phosphorylation (Fig. 4A). We hypothesized that introduction of a phosphate group at the critical -3 and $-5 / 6$ positions could substitute for essential acidic residues to promote binding to the SNX27 PDZ domain.

Phosphorylation of $\beta_{2} \mathrm{AR}$ resulted in two antagonistic effects depending on the location; phosphorylation of $\mathrm{Ser}^{-6}$ or $\mathrm{Thr}^{-5}$ increases binding affinity (from $K_{\mathrm{d}}>200 \mu \mathrm{M}$ to $\sim 94 \mu \mathrm{M}$ and $118 \mu \mathrm{M}$ respectively), while in contrast phosphorylating $\mathrm{Ser}^{-2}$ abolishes interaction, presumably by causing a steric clash with the SNX27 His114 side chain (Fig. 4B; see also Fig. 3C). This is in line with our results

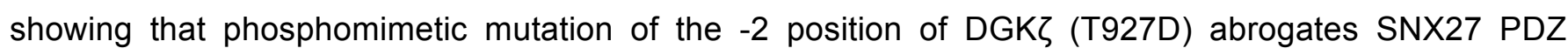
binding (Fig. 3D). Additional retromer engagement improved $\beta_{2} A R-p S^{-6}$ binding from $94 \mu \mathrm{M}$ to $17 \mu \mathrm{M}$, demonstrating again that allostery can turn $\beta_{2} A R$ into a significantly more potent ligand (Fig. 4B, Table S1).

To analyse the effect of phosphorylation on SNX27 association we performed NMR titrations of ${ }^{15} \mathrm{~N}$-labeled SNX27 PDZ domain with $\beta_{2} A R, \beta_{2} A R-p S^{-2}$ and $\beta_{2} A R-p S^{-6}$ peptides (Fig. 4C; Fig. S1). Based upon the backbone resonance assignments performed previously ${ }^{8}$, residues that showed the largest changes in chemical shifts were mapped onto the SNX27 PDZ surface. Both native $\beta_{2} A R$ and $\beta_{2} A R-p S^{-2}$ peptides associated only weakly with the SNX27 PDZ binding pocket, and only at the site where the Cterminal carboxyl group is coordinated. In contrast, $\beta_{2} \mathrm{AR}_{\mathrm{R}} \mathrm{pS}^{-6}$ titration strongly affected residues located around the entire extent of the PDZbm interacting surface (Fig. 4C). This supports the idea that negatively-charged residues and phosphorylation of upstream sequences promotes the coordinated binding of peptides to the extended binding groove. Interestingly, residues in the $\beta$-hairpin of SNX27 that binds to VPS $26{ }^{8}$ also appear to be affected by $\beta 2 A R-p S^{-6}$ binding, suggesting a link between the VPS 26 and peptide binding sites that may relate to the allosteric coupling. Altogether this data suggests 
phosphorylation of $\mathrm{Ser}^{-6} / \mathrm{Ser}^{-5}$ or $\mathrm{Ser}^{-2}$ provide regulatory switches to respectively trigger or prevent SNX27-retromer trafficking of the $\beta_{2} A R$.

\section{Phosphorylation regulates SNX27 binding to NMDA but not AMPA glutamate receptors}

NMDA and AMPA receptors in neurons control glutamatergic synaptic transmission ${ }^{32}$, and their neuronal trafficking is strongly influenced by phosphorylation of their intracellular domains ${ }^{33,34}$. SNX27retromer co-localises with glutamate receptors in neuronal post-synaptic densities ${ }^{12,16,17}$ (Fig. 5A), and forms complexes with these receptors by co-immunoprecipitation. We also observe proximal localisation with GluN2B in dendrites using proximity ligation assay (PLA) (Fig. S2). Similar to $\beta_{2} A R$, glutamate receptors are actively phosphorylated at their C-terminus in vivo (Fig. 4A), and we sought to test whether phosphorylation may provide a regulatory mechanism controlling SNX27 interaction.

The PDZbm from the NMDAR subunit GluN1 interacts only weakly with SNX27 in its native form, but double phosphorylation on $\mathrm{Ser}^{-3}$ and $\mathrm{Ser}^{-5}$ greatly improves binding (Fig. 5B; Table S1), suggesting phosphorylation of GluN1 at these sites can mimic the electrostatic plug for SNX27 Arg58. Similarly, phosphorylation of GluN2B at $\mathrm{Ser}^{-5}$ and $\mathrm{Ser}^{-6}$ also confers a much higher affinity, confirming that phosphorylation mimics the contribution of Asp/Glu residues found in constitutively high affinity ligands (Fig. 5B; Table S1). Retromer association enhances binding to both peptides (Fig. 5B, Table S1). To confirm the binding of NMDARs and SNX27 in cells, we performed pulldowns of myc-SNX27 with GSTGluN1 or GST-GluN2B C-terminal tail fusion proteins harbouring phosphorylation defective (AA) or mimetic (DD) mutations. Compared to the wild-type GluN2B, the phosphomimetic mutation at $-5 /-6$ significantly improves binding. The effect is even more pronounced for GluN1, where binding is hard to detect for both the wild-type protein and phosphodefective mutant, but is dramatically enhanced by phosphomimetic mutation at $-3 /-5$ sites (Fig. 5C). This supports the idea that certain 'weak' sequences such as GluN1 lacking an acidic residue at -3 are unable to interact with SNX27 unless they are phosphorylated, while cargos that possess this acidic residue such as GluN2B can be significantly enhanced by phosphorylation. The identities of the -3 and $-5 /-6$ residues in these cargos are strictly conserved, implying they are functionally important (Fig. 5D) ${ }^{35}$.

Previous studies have suggested a role for SNX27-retromer in transport of the AMPARs ${ }^{12,16,17}$. However, we do not detect significant affinity for AMPAR peptides in native or phosphorylated states (Fig. S3). GluA1 possesses a type I PDZ motif ([ST]X $\phi)$, but lacks the acidic side-chain at the -3 site and does not bind strongly to the SNX27 PDZ domain even in the presence of VPS26. GluA2 has a type II motif $(\phi \times \phi)$ and also does not associate significantly with SNX27, even after phosphorylation of Ser ${ }^{-3}$ or $\mathrm{Tyr}^{-7}$. Based on structural considerations it is clear that although GluA1 may be physically able to associate, albeit weakly because of the lack of the acidic -3 side-chain, the binding of GluA2 is precluded because it lacks the key Ser/Thr ${ }^{-2}$ side-chain required for interacting with SNX27 His114. In the context of overall postsynaptic receptor trafficking we propose that NMDARs represent vastly more efficient cargos than AMPARs for SNX27-mediated recycling. 


\section{Mechanism of SNX27 binding to phosphorylated PDZbms}

Based on our results with $\beta 2 A R$ and NMDARs, we scanned known SNX27 binders for other sequences potentially enhanced by phosphorylation at the -3 and -5 or -6 positions and tested two of these; the sorting nexin SNX14 ${ }^{9}$ and the GPCR 5- $\mathrm{HT}_{4(\mathrm{a})} \mathrm{R}{ }^{19}$ (Fig. 6A; Table S1). A phosphomimetic SNX14 sequence showed significant binding to SNX27 as expected $\left(K_{d} \sim 9 \mu \mathrm{M}\right)$, while a phosphorylated version of the $5-\mathrm{HT}_{4(\mathrm{a})} \mathrm{R}$ bound to SNX27 with an affinity nearly 4 fold greater than the native sequence and was enhanced to a $K_{d}$ of $1.2 \mu \mathrm{M}$ by the allosteric effect of VPS26 binding. This further supports the role of PDZbm phosphorylation in conferring high affinity interaction with SNX27 by mimicking key acidic residues. To confirm the mechanism of phosphorylation-enhanced binding we determined the high resolution crystal structure of the complex between the SNX27 PDZ domain and phosphorylated 5$\mathrm{HT}_{4(\mathrm{a})} \mathrm{R}$ PDZbm (Fig. 6B; Table S2). The crystal structure shows that the phosphoserine $\mathrm{pSer}^{-5}$ cooperates with the acidic $\mathrm{Glu}^{-3}$ side chain to enable the clamping of SNX27 Arg58 as done by glutamates and aspartates of other PDZbms (Fig. 6B).

We next confirmed the structural basis for enhanced SNX27 affinity via phosphorylation of the critical PDZbm -3 side-chain. We solved the crystal structures of two different peptide complexes; a mutated version of the PTHR PDZbm where $\mathrm{Glu}^{-3}$ was artificially altered to phosphoserine, and the leucine-rich repeat-containing protein 3B (LRRC3B; identified in bioinformatics analyses discussed below (Fig. 6D and 6E; Table S2)). The phosphorylated PTHR PDZbm has a similar affinity to the native peptide (not shown), indicating $\mathrm{pSer}^{-3}$ establishes similar functional bonds to maintain the previously described Arg58 plug. Phosphorylation of LRRC3B dramatically increases affinity compared to the native sequence as expected (Fig. 6C). The two co-crystal structures reveal at ultra high resolution the mechanism of interaction, where the phosphate modification is intimately coordinated by hydrogen bonds and electrostatic interactions with Asn56, Arg58 and Ser82. The precision of our data also confirms that while the four terminal residues of the PDZbm adopt a precise orientation to satisfy the constraints of these bonds, upstream residues can adapt their backbone conformation to promote pairing with Arg58. In addition, Arg58 adopts a flipped conformation to accommodate $\mathrm{pSer}^{-5}$ in the $5-\mathrm{HT} 4_{(\mathrm{a})} \mathrm{R}$ peptide (Fig. 6F). These structures provide a clear description of how phosphorylated residues at -3 and -5 positions mimic acidic residues present in constitutively high affinity SNX27 ligands.

A question that arises is whether phosphorylation may be a more general signal for enhancing PDZ domain interactions. Sequence alignments of PDZ domains suggest that only those of NHERF1 and NHERF2 possess side-chains with potential to coordinate negative charges similar to the Asn56/Arg58/Ser82 triplet in SNX27 ${ }^{36}$. For example NHERF1 PDZ2 has His/His/Arg in the analogous sites (Fig. S4A and B). CFTR is a validated binder of both NHERF1 and NHERF2 ${ }^{37}$, and we see robust interaction of NHERF1 PDZ2 domain with the CFTR control by ITC (Fig. S4C; Table S1). We also see similar binding of CFTR to SNX27, and SNX27 was recently identified as a high confidence binder in CFTR proteomics ${ }^{38}$. However, when we tested either GluN2B (Fig. S4C), GluN1 or LRRC3B (not 
shown) in either their native or phosphorylated states, we could not detect any binding by NHERF1. This suggests that phosphorylated PDZbms bind preferentially to SNX27.

\section{Bioinformatic discovery of SNX27 interacting proteins}

Combining our data with analysis of previously identified SNX27-interacting proteins (Table S3) we can define three major classes of PDZbm-containing ligands, where the identities of the last seven amino acids of the protein influence binding affinity. Acidic residues or phosphorylated side-chains at the $-3,-5$ (and to a lesser extent -6) positions consistently enhance SNX27 affinity (Fig. S5; Table S1). Modulating these three positions thus yields predictable classes of SNX27 binders (Fig. 7A). Class 1a peptides are strong interactors that clamp Arg58 in SNX27, and are likely to constitutively associate under physiological conditions. Class $2 a$ binders possess motifs where binding is enhanced by phosphorylation at the -5 position, whereas Class $2 b$ and $2 c$ peptides contain potential sites of phosphorylation at the -3 position that is critical for SNX27 binding. Our data also suggests that additional acidic or phosphorylated residues upstream at the -6 position positively influence association through localised electrostatic interactions with SNX27 (Classes $1 b, 2 b$ and $2 c$ ). Class $3 a$ binders are ligands that bind relatively weakly to SNX27. Finally, Class $3 b$ binders are those with Thr or Ser at the -3 position that are unlikely to bind at all to SNX27 without phosphorylation.

These newly defined consensus motifs allowed us to search the human genome, and identify 432 putative ligand and cargo molecules that possess the molecular signatures required to bind the SNX27retromer complex (Fig. S6, Table S4). A number of these were previously identified, but our bioinformatics imply that most of the SNX27 PDZ interactome still remains unexplored. Correlating with our predictions, the greatest proportion of previously identified ligands fall within the classes we predict will have the highest affinity for the SNX27-retromer complex. To begin to test these predictions we analysed the levels of several receptors in HeLa cells with SNX27 deleted by CRISPR/Cas9. Consistent with defective recycling and lysosomal turnover the levels of ATP7A and PDGFR $\beta$ were reduced in SNX27 knockout cells, as was the sorting nexin SNX14 (Fig. 7B). SNX14 is member of a distinct sorting nexin sub-family localised at endosomes at steady state ${ }^{39,40}$, but whether it undergoes plasma membrane trafficking like other transmembrane cargos or is simply stabilised at endosomal compartments by interaction with SNX27 is unclear. Interestingly, the solute carrier EAAT1/SLC1A3 does not appear to be degraded in SNX27 knockout cells, although a reduction was previously observed in proteomics studies following siRNA of SNX27 ${ }^{9}$. 


\section{DISCUSSION}

We have investigated the molecular aspects of cargo and accessory protein binding to the endosomal trafficking complex SNX27-retromer. The combined action of Arg58, Asn56 and Ser82 in SNX27 provides a key binding site for a negatively charged acidic side-chain at the -3 site of ligand PDZbms that is essential for interaction. This correlates with proteomics data suggesting that the large majority of identified SNX27 interactors that possess a PDZ motif incorporate an acidic residue at the -3 position ${ }^{9}$. In addition Arg58 can accommodate binding to an adjacent acidic side-chain in the peptide -5 position, in an arrangement we refer to as the 'electrostatic plug'. To a lesser extent acidic side-chains in the -6 position also promote SNX27 interaction through general electrostatic interactions. Previous work by Balana and colleagues on the G-protein inwardly rectifying potassium channels support these findings ${ }^{14}$. Our data shows that retromer allosterically enhances binding to SNX27 for all cargo and ligand PDZbms, and it is also worth noting that association within the context of the membrane bilayer, coupled to lipid interactions of the SNX27 PX and FERM domains ${ }^{41,42}$, will act to coordinate their physiological association at the endosome. It should be noted that many proteins identified in SNX27 proteomics do not possess PDZ motifs ${ }^{9}$, some of these possess NPXY sequences ${ }^{43}$, while others may be recruited through yet to be determined mechanisms.

The most striking observation is that the binding of many PDZbms to SNX27 is enhanced by phosphorylation. Our data shows phosphate groups add negative charges that when placed in $-3,-5$ and -6 positions promote SNX27-retromer association. In contrast, phosphorylating the -2 side-chain inhibits binding as the phosphate group is sterically precluded. Phosphorylation of the -2 residue in Type 1 PDZbms of several proteins, including NMDARs and $\beta_{2} A R^{44-52}$, has previously been found to negatively regulate binding to other PDZ domains. Only one previous structure of a PDZ domain bound to a phosphorylated peptide has been determined, describing the complex between the PDZ domain of TIAM1 and a type 2 PDZbm from syndecan-1 Tyr-phosphorylated at the -1 position ${ }^{53}$. Binding studies have also been performed with the ZO-1 PDZ domain binding type 2 PDZbm from claudin-2 Tyrphosphorylated at the -6 residue ${ }^{54}$. In the former case phosphorylation alters the tyrosine binding conformation but has no effect on binding affinity, while in the latter case phosphorylation inhibits the interaction. Although inhibitory effects of phosphorylation are well established, to the best of our knowledge a positive role in PDZ interactions has not previously been observed. The dramatic enhancement of PDZbm binding to SNX27 suggests an important and previously unidentified role for post-translational modification in promoting PDZ domain interactions.

Structural studies demonstrate how phosphorylation mimics the electrostatic plug centred on Arg58 to potentiate SNX27 binding. The consistent enhancement of binding by phosphorylation of highly conserved sequences indicates that it almost certainly represents a functional signal for regulating cargo transport along the SNX27-retromer pathway. The PhosphositePlus Database (phosphosite.org) confirms that many of the predicted ligands are phosphorylated at the required sites, although the signals leading to these modifications remain unknown (e.g. Fig. 4A). Many transmembrane cargos are 
modified at their C-termini by kinases and phosphatases ${ }^{49,55}$, and an important question will be to determine which enzymes are involved in regulating SNX27-retromer interactions, and what impact these modifications have on endosomal transport. For example, GRK2 phosphorylates $\beta_{2} A R$ at both Ser 2 and Ser $^{-6}$ upon agonist stimulation ${ }^{56}$, although it is unknown whether this particular kinase modulates endocytic recycling.

Our results delineate three broad classes of SNX27 PDZbm ligands; strongly and weakly associated cargoes with and without acidic side-chains respectively at the $-3,-5$ and -6 positions, and others whose association is controlled by phosphorylation. We speculate that this 'electrostatic code' defines a specificity for SNX27-retromer association that will determine the efficiency and timing of endosomal recycling; some cargos being recycled constitutively, and others being recycled efficiently only under certain conditions of cellular stimulation causing their phosphorylation. A pathway analysis of the molecules we identified in the SNX27 interactome supports a prominent role for SNX27-retromer in trafficking cargoes involved in synaptic function and cell signalling as suggested by previous studies ${ }^{9}$ (Fig. 7C; Table S4; Table S5). Surprisingly however, the largest subset of putative ligands are members of the metabolite transporters/solute carrier family, transmembrane proteins whose trafficking is very poorly understood, although they are of major importance in human physiology ${ }^{57}$. Many putative SNX27 binding proteins are of unknown biological function, suggesting novel discoveries regarding SNX27's role in endosomal transport and signalling are yet to come. It is also worth noting that some pathogens can interact with SNX27 through similar mechanisms, and are likely to compete with and perturb normal transport processes ${ }^{58,59}$.

In summary, our study describes an adaptable molecular code controlling SNX27-retromer recruitment of many different cargos including GPCRs, ion channels and solute carriers, as well as soluble signalling and regulatory proteins. In a broader context our data provides new mechanistic insights into how PDZ domains can fine-tune the dynamics of protein interaction networks. 


\section{METHODS}

Methods and any associated references are available in the online version of the paper. 


\section{Accession Codes}

Structural data is deposited in the protein data bank (PDB) under accession numbers 5ELQ, 5EM9, 5EMA and 5EMB for the SNX27 PDZ domain in complex with PDZbm peptides from DGK $\zeta$, and phosphorylated $5-\mathrm{HT}_{4(\mathrm{a})} \mathrm{R}$, LRR3CB and PTHR(pSer) respectively. Raw diffraction images are available at the University of Queensland eSpace (https://espace.library.uq.edu.au).

\section{Author contributions}

All X-ray crystallography, bioinformatics and ITC studies were carried out by TC, MC and BMC with help from BP. NMR experiments were performed by TC and CM. Cell biology experiments were performed by MTL, IM, ASMC, NJP, JW, ZY, MCK and VA. BMC and RDT conceived the project, and BMC coordinated the project and wrote the paper together with TC, IM, RDT, NP and VA.

\section{Acknowledgements}

The authors would like to acknowledge support from the staff and facilities of the University of Queensland Remote Operation Crystallization and X-ray (UQ ROCX) facility, and the Australian Synchrotron. We thank Bruno Madio for assistance with sequence alignment, and Mehdi Mobli for assistance with NMR spectroscopy. This work is supported by funds from the Australian Research Council (ARC) (DP0985029), National Health and Medical Research Council (NHMRC) (APP1042082, APP1058734 and APP1078280), UWA-UQ Bilateral Research Collaboration Award (to NJP, RDT, BMT) and the John T. Reid Charitable Trusts (to VA). CM was supported by a University of Queensland Postdoctoral Fellowship, RDT is supported by an NHMRC Senior Research Fellowship (APP1041929), and BMC is supported by an NHMRC Career Development Fellowship (APP1061574) and previously held an ARC Future Fellowship (FT100100027). MT receives an FPI fellowship from the Spanish Ministry of Economy and Competitiveness. IM receives support from the Spanish Ministry of Economy and Competitiveness (BFU2013-47640-P), and the Madrid regional government (IMMUNOTHERCAM Consortium S2010/BMD-2326). 


\section{REFERENCES}

1. Kennedy, M.B. Signal-processing machines at the postsynaptic density. Science 290, 750-4 (2000).

2. Zhang, M. \& Wang, W. Organization of signaling complexes by PDZ-domain scaffold proteins. Acc Chem Res 36, 530-8 (2003).

3. Kim, E. \& Sheng, M. PDZ domain proteins of synapses. Nat Rev Neurosci 5, 771-81 (2004).

4. Hung, A.Y. \& Sheng, M. PDZ domains: structural modules for protein complex assembly. J Biol Chem 277, 5699-702 (2002).

5. Stiffler, M.A. et al. PDZ domain binding selectivity is optimized across the mouse proteome. Science 317, 364-9 (2007).

6. Hall, R.A. et al. The beta2-adrenergic receptor interacts with the $\mathrm{Na}+\mathrm{H}+$-exchanger regulatory factor to control $\mathrm{Na}+/ \mathrm{H}+$ exchange. Nature 392, 626-30 (1998).

7. Ye, F. \& Zhang, M. Structures and target recognition modes of PDZ domains: recurring themes and emerging pictures. Biochem J 455, 1-14 (2013).

8. Gallon, M. et al. A unique PDZ domain and arrestin-like fold interaction reveals mechanistic details of endocytic recycling by SNX27-retromer. Proc Natl Acad Sci U S A 111, E3604-13 (2014).

9. Steinberg, F. et al. A global analysis of SNX27-retromer assembly and cargo specificity reveals a function in glucose and metal ion transport. Nat Cell Biol 15, 461-71 (2013).

10. Lauffer, B.E. et al. SNX27 mediates PDZ-directed sorting from endosomes to the plasma membrane. J Cell Biol 190, 565-74 (2010).

11. Temkin, P. et al. SNX27 mediates retromer tubule entry and endosome-to-plasma membrane trafficking of signalling receptors. Nat Cell Biol 13, 715-21 (2011).

12. Choy, R.W. et al. Retromer mediates a discrete route of local membrane delivery to dendrites. Neuron 82, 55-62 (2014).

13. Chan, A. et al. Sorting Nexin 27 couples PTHR trafficking to retromer for signal regulation in osteoblasts during bone growth. Mol. Biol. Cell. Epub(2016).

14. Balana, B. et al. Mechanism underlying selective regulation of $\mathrm{G}$ protein-gated inwardly rectifying potassium channels by the psychostimulant-sensitive sorting nexin 27. Proc Natl Acad Sci U S A 108, 5831-6 (2011).

15. Lunn, M.L. et al. A unique sorting nexin regulates trafficking of potassium channels via a PDZ domain interaction. Nat Neurosci 10, 1249-59 (2007).

16. Hussain, N.K., Diering, G.H., Sole, J., Anggono, V. \& Huganir, R.L. Sorting Nexin 27 regulates basal and activity-dependent trafficking of AMPARs. Proc Natl Acad Sci U S A 111, 11840-5 (2014).

17. Loo, L.S., Tang, N., Al-Haddawi, M., Dawe, G.S. \& Hong, W. A role for sorting nexin 27 in AMPA receptor trafficking. Nat Commun 5, 3176 (2014).

18. Wang, X. et al. Loss of sorting nexin 27 contributes to excitatory synaptic dysfunction by modulating glutamate receptor recycling in Down's syndrome. Nat Med 19, 473-80 (2013).

19. Joubert, L. et al. New sorting nexin (SNX27) and NHERF specifically interact with the 5-HT4a receptor splice variant: roles in receptor targeting. J Cell Sci 117, 5367-79 (2004). 
20. Lee, S., Chang, J. \& Blackstone, C. FAM21 directs SNX27-retromer cargoes to the plasma membrane by preventing transport to the Golgi apparatus. Nat Commun 7, 10939 (2016).

21. Muhammad, A. et al. Retromer deficiency observed in Alzheimer's disease causes hippocampal dysfunction, neurodegeneration, and Abeta accumulation. Proc Natl Acad Sci U S A 105, 7327-32 (2008).

22. Vilarino-Guell, C. et al. VPS35 mutations in Parkinson disease. Am J Hum Genet 89, 162-7 (2011).

23. Wen, L. et al. VPS35 haploinsufficiency increases Alzheimer's disease neuropathology. J Cell Biol 195, 765-79 (2011).

24. Zimprich, A. et al. A mutation in VPS35, encoding a subunit of the retromer complex, causes lateonset Parkinson disease. Am J Hum Genet 89, 168-75 (2011).

25. Wang, $X$. et al. Sorting Nexin 27 Regulates Abeta Production through Modulating gammaSecretase Activity. Cell Rep 9, 1023-33 (2014).

26. Damseh, N. et al. A defect in the retromer accessory protein, SNX27, manifests by infantile myoclonic epilepsy and neurodegeneration. Neurogenetics (2015).

27. Tsika, E. et al. Parkinson's disease-linked mutations in VPS35 induce dopaminergic neurodegeneration. Hum Mol Genet (2014).

28. Vardarajan, B.N. et al. Identification of Alzheimer disease-associated variants in genes that regulate retromer function. Neurobiol Aging 33, 2231 e15-2231 e30 (2012).

29. Rincon, E. et al. Translocation dynamics of sorting nexin 27 in activated T cells. J Cell Sci 124, 776-88 (2011).

30. Rincon, E. et al. Proteomics identification of sorting nexin 27 as a diacylglycerol kinase zetaassociated protein: new diacylglycerol kinase roles in endocytic recycling. Mol Cell Proteomics 6, 107387 (2007).

31. Sowa, M.E., Bennett, E.J., Gygi, S.P. \& Harper, J.W. Defining the human deubiquitinating enzyme interaction landscape. Cell 138, 389-403 (2009).

32. Okabe, S. Molecular anatomy of the postsynaptic density. Mol Cell Neurosci 34, 503-18 (2007).

33. Wang, J.Q. et al. Roles of subunit phosphorylation in regulating glutamate receptor function. Eur J Pharmacol 728, 183-7 (2014).

34. Lussier, M.P., Sanz-Clemente, A. \& Roche, K.W. Dynamic Regulation of N-Methyl-d-aspartate (NMDA) and alpha-Amino-3-hydroxy-5-methyl-4-isoxazolepropionic Acid (AMPA) Receptors by Posttranslational Modifications. J Biol Chem 290, 28596-603 (2015).

35. Ryan, T.J., Emes, R.D., Grant, S.G. \& Komiyama, N.H. Evolution of NMDA receptor cytoplasmic interaction domains: implications for organisation of synaptic signalling complexes. BMC Neurosci $\mathbf{9}, 6$ (2008).

36. Sakarya, O. et al. Evolutionary expansion and specialization of the PDZ domains. Mol Biol Evol 27, 1058-69 (2010).

37. Cushing, P.R., Fellows, A., Villone, D., Boisguerin, P. \& Madden, D.R. The relative binding affinities of PDZ partners for CFTR: a biochemical basis for efficient endocytic recycling. Biochemistry 47, 10084-98 (2008). 
38. Pankow, S. et al. $\triangle$ F508 CFTR interactome remodelling promotes rescue of cystic fibrosis. Nature Epub(2015).

39. Akizu, N. et al. Biallelic mutations in SNX14 cause a syndromic form of cerebellar atrophy and lysosome-autophagosome dysfunction. Nat Genet 47, 528-34 (2015).

40. Mas, C. et al. Structural basis for different phosphoinositide specificities of the PX domains of sorting nexins regulating G-protein signaling. J Biol Chem 289, 28554-68 (2014).

41. Ghai, R. et al. Phox homology band 4.1/ezrin/radixin/moesin-like proteins function as molecular scaffolds that interact with cargo receptors and Ras GTPases. Proc Natl Acad Sci U S A 108, 7763-8 (2011).

42. Ghai, R. et al. Phosphoinositide binding by the SNX27 FERM domain regulates its localization at the immune synapse of activated T-cells. J Cell Sci 128, 553-65 (2015).

43. Ghai, R. et al. Structural basis for endosomal trafficking of diverse transmembrane cargos by PXFERM proteins. Proc Natl Acad Sci U S A 110, E643-52 (2013).

44. $\quad$ Cao, T.T., Deacon, H.W., Reczek, D., Bretscher, A. \& von Zastrow, M. A kinase-regulated PDZdomain interaction controls endocytic sorting of the beta2-adrenergic receptor. Nature 401, 286-90 (1999).

45. Choi, J. et al. Phosphorylation of stargazin by protein kinase A regulates its interaction with PSD95. J Biol Chem 277, 12359-63 (2002).

46. Chung, H.J., Huang, Y.H., Lau, L.F. \& Huganir, R.L. Regulation of the NMDA receptor complex and trafficking by activity-dependent phosphorylation of the NR2B subunit PDZ ligand. $J$ Neurosci $\mathbf{2 4}$, 10248-59 (2004).

47. Chung, H.J., Xia, J., Scannevin, R.H., Zhang, X. \& Huganir, R.L. Phosphorylation of the AMPA receptor subunit GluR2 differentially regulates its interaction with PDZ domain-containing proteins. $J$ Neurosci 20, 7258-67 (2000).

48. Cohen, N.A., Brenman, J.E., Snyder, S.H. \& Bredt, D.S. Binding of the inward rectifier K+ channel Kir 2.3 to PSD-95 is regulated by protein kinase A phosphorylation. Neuron 17, 759-67 (1996).

49. Sanz-Clemente, A., Matta, J.A., Isaac, J.T. \& Roche, K.W. Casein kinase 2 regulates the NR2 subunit composition of synaptic NMDA receptors. Neuron 67, 984-96 (2010).

50. Tanemoto, M., Fujita, A., Higashi, K. \& Kurachi, Y. PSD-95 mediates formation of a functional homomeric Kir5.1 channel in the brain. Neuron 34, 387-97 (2002).

51. Tian, Q.B. et al. Interaction of LDL receptor-related protein 4 (LRP4) with postsynaptic scaffold proteins via its $\mathrm{C}$-terminal PDZ domain-binding motif, and its regulation by $\mathrm{Ca} /$ calmodulin-dependent protein kinase II. Eur J Neurosci 23, 2864-76 (2006).

52. Lee, H.J. \& Zheng, J.J. PDZ domains and their binding partners: structure, specificity, and modification. Cell Commun Signal 8, 8 (2010).

53. Liu, X., Shepherd, T.R., Murray, A.M., Xu, Z. \& Fuentes, E.J. The structure of the Tiam1 PDZ domain/ phospho-syndecan 1 complex reveals a ligand conformation that modulates protein dynamics. Structure 21, 342-54 (2013).

54. Nomme, J. et al. Structural Basis of a Key Factor Regulating the Affinity between the Zonula Occludens First PDZ Domain and Claudins. J Biol Chem 290, 16595-606 (2015). 
55. Zhang, N. et al. Phosphorylation of Synaptic Vesicle Protein 2A at Thr84 by Casein Kinase 1 Family Kinases Controls the Specific Retrieval of Synaptotagmin-1. J Neurosci 35, 2492-507 (2015).

56. Nobles, K.N. et al. Distinct phosphorylation sites on the beta(2)-adrenergic receptor establish a barcode that encodes differential functions of beta-arrestin. Sci Signal 4, ra51 (2011).

57. Cesar-Razquin, A. et al. A Call for Systematic Research on Solute Carriers. Cell 162, 478-87 (2015).

58. Belotti, E. et al. The human PDZome: a gateway to PSD95-Disc large-zonula occludens (PDZ)mediated functions. Mol Cell Proteomics 12, 2587-603 (2013).

59. Pim, D., Broniarczyk, J., Bergant, M., Playford, M.P. \& Banks, L. A Novel PDZ Domain Interaction Mediates the Binding between Human Papillomavirus 16 L2 and Sorting Nexin 27 and Modulates Virion Trafficking. J Virol 89, 10145-55 (2015). 


\section{FIGURE LEGENDS}

Figure 1. High affinity binding of PTHR to SNX27-retromer requires upstream acidic residues (A) Cartoon illustration of the endosomal recycling pathway mediated by the SNX27-retromer complex. Endocytosed cargo molecules (often stimulated by ligand binding and activation) associate with SNX27 within the endosome, are sorted into tubulovesicular membrane domains through allosteric coupling with the retromer complex, and return to the cell surface, avoiding degradation within the late endosome/lysosome compartment. (B) Structure of the SNX27 PDZ domain bound to PDZbm peptides from Kir3.3 potassium channel (green; PDD ID 3QDO) ${ }^{14}$ and PTHR (yellow; PDB ID 4Z8J) ${ }^{13}$. The peptides bind to the SNX27 PDZ domain canonical binding groove in a $\beta$-strand conformation. Two glutamate residues in -3 and -5 positions interact with a basic patch to potentially stabilise the complex. (C) Mutagenesis of the PTHR PDZbm and binding to the SNX27 PDZ domain (data marked with an asterisk $\left(^{*}\right)$ from ${ }^{13}$ are shown for comparison. (D) Colocalisation of SNX27 (green) with internalised PTH-PTHR ligand-receptor complexes following agonist stimulation with $100 \mathrm{nM} \mathrm{PTH}^{\mathrm{TMR}}$ (magenta) for 15 minutes in HEK293 cells stably expressing PTHR. Bar, $10 \mu \mathrm{m}$. Correlative line-scan analysis of SNX27 and PTH ${ }^{\mathrm{TMR}}$ intensity profiles as shown in the merged inset. Bar, $1 \mu \mathrm{m}$. (E) PTHR constructs containing C-terminal PDZbms as indicated in panel C show differential endosomal recruitment with SNX27 following agonist stimulation and endocytosis. Deletion of the PDZbm or alteration of the acidic plug causes a defect in SNX27 association. Bar, $1 \mu \mathrm{m}$. (F) Colocalisation analysis (Pearson's coefficient, $R r$ ) of SNX27 with internalised PTH ${ }^{-T M R}$ at 15 minutes. Values are mean \pm SEM $n=10 .{ }^{* *} p<0.01,{ }^{* * *} p<$ 0.001 .

Figure 2. Electrostatic interactions established by a subset of PDZbms promote high affinity binding to SNX27-retromer

(A) ITC experiments with the SNX27 PDZ domain alone (black) or in complex with VPS26 (blue) show binding to two ligands of the SNX27-retromer complex $\beta_{2} A R$ and DGKל. The H114A mutation in SNX27 is a negative control that disrupts binding to PDZbms (orange). Artificial introduction of acidic residues in $\beta_{2} A R$ at the -5 and -6 positions or removal of the analogous residues in DGK binding to the SNX27 PDZ domain respectively (purple). Raw data and integrated and normalised data are shown at top and bottom respectively. (B) ITC binding curve of the KIDINS220 PDZbm peptide interacting with SNX27. (C) ITC binding curves of the PHLPP1 and PHLPP2 PDZbm peptides interacting with SNX27.

\section{Figure 3. Crystal structure of the SNX27 PDZ domain in complex with the DGKろ PDZbm peptide}

(A) The $1.1 \AA$ resolution crystal structure of the SNX27 PDZ domain is shown in ribbon representation, the bound DGKל PDZbm peptide is shown in brown stick representation, and its refined $2 \mathrm{~F}_{\mathrm{o}}-\mathrm{F}_{\mathrm{c}}$ electron density contoured at $2 \sigma$ is shown in magenta. (B) Comparison of the DGK $\xi$ peptide (brown) with Kir3.3 (green) ${ }^{14}$ and PTHR (yellow) ${ }^{13}$. Each of these peptides make electrostatic and hydrogen bonds to 
Arg58 and Asn56 of SNX27 through the conserved $\mathrm{Glu}^{-3}$ side chains. The Asp ${ }^{-5}$ of DGK $\zeta$ and corresponding $\mathrm{Glu}^{-5}$ of PTHR also make stable contact to Arg58 of SNX27 forming what we refer to as the electrostatic plug. (C) Surface representation of the SNX27 PDZbm binding groove (centre panel), highlighting the significant positive electrostatic potential of the binding surface (blue). An expanded view of the variable side-chains at the peptide -4 position for DGK (brown), PTHR (yellow) and Kir3.3 (green) is shown in the left panel. An expanded view of the canonical PDZbm C-terminal triplet for DGK $\zeta$ is shown in the right panel. This highlights the hydrogen bonding of the C-terminal carboxyl group to SNX27 main chain atoms, and the hydrogen bond interaction of $\mathrm{Thr}^{-2}$ to SNX27 His114. (D) GFP-DGK $\xi$ wild type and mutant constructs expressed in Jurkat T-cells were analysed for binding to SNX27 by GFP-trap immunoprecipitation and immunoblotting with the indicated antibodies.

Figure 4. Phosphorylation of upstream residues in the $\beta_{2} A R$ PDZbm promote specific association with SNX27

(A) Sequence alignment of synaptic plasticity receptors cytoplasmic C-termini highlight the presence of serine/threonine residues which we predict promote or disrupt (red and blue respectively) binding to SNX27 and are phosphorylated in vivo according to the PhosphoteSite database (grey boxes). (B) (Left) ITC experiments show binding of $\beta_{2} A R$ phosphorylated peptides (colours) compared to the wild type receptor (black). Thermodynamic parameters of binding are given in Table S1. Phosphorylation at -5 or 6 positions significantly increases affinity and enthalpy of binding. Phosphorylation at the -2 position abolishes interaction. (Right) VPS26 engagement improves binding parameters of both wild type (blue) and $\beta_{2} A R$ peptides phosphorylated at the -6 position (cyan). (C) According to the NMR titration of $\beta_{2} A R$, $\beta_{2} A R-p S^{-2}$ and $\beta_{2} A R-p S^{-6}$ (see ${ }^{1} \mathrm{H}^{-15} \mathrm{~N}$ HSQC spectra in Fig. S2), residues showing significant line broadening and change of chemical shift upon peptide addition are mapped in blue/cyan, pink/purple and red/orange respectively onto the SNX27 PDZ domain surface (a modelled DGK $\zeta$ peptide displayed in purple sticks highlights the elements involved in binding PDZbms).

\section{Figure 5. Phosphorylation of the GluN1 and GluN2B subunits of NMDARs triggers interaction with SNX27}

(A) Hippocampal neurons (15 days in vitro) were transfected with GFP-GluN2B (green) and myc-SNX27 (magenta) for 48 hours. Arrowheads indicate colocalisation of these two proteins in dendritic spines (white). Scale bars, $50 \mu \mathrm{m}$ (top) and $5 \mu \mathrm{m}$ (bottom). (B) Comparison of the binding profile of both normal and phosphorylated NMDAR PDZbm peptides to free or VPS26-engaged SNX27 PDZ domain. Peptide sequences used for each experiment are indicated on the right. (C) HEK293 cells were co-transfected with myc-SNX27 and GST-GluN1 or GST-GluN2B C-terminal tails, either WT, PDZ mutant, phosphodefective (AA) or phosphomimetic (DD) mutant and pull-down experiments from cell lysates were performed with GST proteins as bait. (D) Amino acid conservation of the PTHR and NMDAR GluN1/N2B PDZbms. PDZbms were aligned with their closest 100 homologs found by BLAST search, 
and the consensus sequence of the alignment based on the C-terminus was extracted for comparison. Alignment was made with CLC sequence viewer.

Figure 6. Crystal structure of the SNX27 PDZ domain in complex with phosphorylated PDZbm peptides

(A) ITC experiments with the SNX27 PDZ domain alone (black and red) or in complex with VPS26 (blue) show binding to $5-\mathrm{HT}_{4(\mathrm{a})} \mathrm{R}, 5-\mathrm{HT}_{4(\mathrm{a})} \mathrm{R}-\mathrm{pS}^{-5}$ and SNX14 (phosphomimetic) peptides. (B) The $1.6 \AA$ resolution crystal structure of the SNX27 PDZ domain is shown in ribbon representation, the bound 5$\mathrm{HT}_{4(\mathrm{a})} \mathrm{R}$ PDZbm peptide is shown in blue stick representation, and its refined $2 \mathrm{~F}_{\mathrm{o}}-\mathrm{F}_{\mathrm{c}}$ electron density contoured at $1.5 \sigma$ is shown in orange. The higher black panel shows a zoom of the Arg58 plug, and the lower black panel shows distance measurements for critical bonds that maintain the peptide bound to the PDZ cavity. (C) ITC experiments of SNX27PDZ binding to native and phosphorylated LRRC3B PDZbms. (D) The crystal structures of the SNX27 PDZ domain (grey) bound to the LRRC3B-pSer ${ }^{-3}$ (violet) and (E) PTHR-pSer ${ }^{-3}$ (green) PDZbm peptides are shown with their refined $2 \mathrm{~F}_{\mathrm{o}}-\mathrm{F}_{\mathrm{c}}$ electron density contoured at $1.5 \sigma$ and $1.8 \sigma$ respectively. (F) The structural alignment of phosphopeptides bound to the SNX27 PDZ domain highlights the contribution of phosphate ions and their mimicry of acidic residues in two separate positions to promote binding to conserved SNX27 residues.

Figure 7. Classification of the SNX27 putative PDZ interactome on the basis of C-terminal sequence

(A) Schematic representation of the SNX27 PDZ binding pocket (brown) and the molecular contacts (orange dashed lines) established with strong PDZ ligands (black). The presence of acidic residues or phosphate headgroups (green) in positions -3/5/6 introduces a negative charge, binding to the SNX27 surface. For all PDZ ligands, phosphorylating the Ser/Thr ${ }^{-2}$ residue disrupts binding to the PDZ domain. The three classes of PDZbms can be sub-categorised and are defined by consensus sequences emerging from our findings (bottom). $\phi=\mathrm{I}, \mathrm{L}, \mathrm{V}, \mathrm{M}$ or F. (B) Steady state levels of putative SNX27 cargos are reduced following SNX27 knockout by CRISPR/Cas9 deletion. Whole cell lysates from control HeLa cells (stably expressing Cas9) and SNX27 knockout cells were probed for several PDZbmcontaining cargos by western blotting. Reduction of PDGFR $\beta$, ATP7A and SNX14 suggests a defect in endosomal transport leading to lysosomal degradation. EAAT1 total levels are apparently unaltered. $\mathrm{Na}^{+} / \mathrm{K}^{+}$-ATPase is a control plasma membrane protein. (C) Schematic representation of the different functions of PDZbms trafficked by SNX27. The 432 putative interacting proteins curated from our human proteome screen were analysed by Gene Ontology (GO) processes, and the top 250 enriched pathways (based on their false discovery rate) were manually classified into functional groups and pictured as spheres. The proteins comprising the network are also annotated based on their molecular function (bottom right histogram) (related to Table S5). 
A

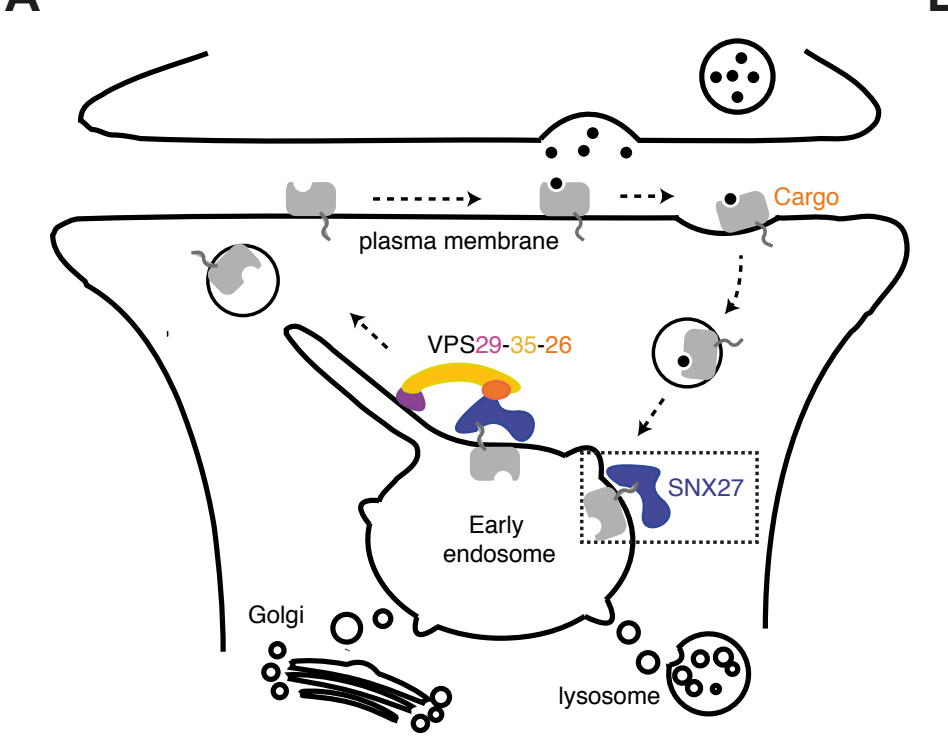

C

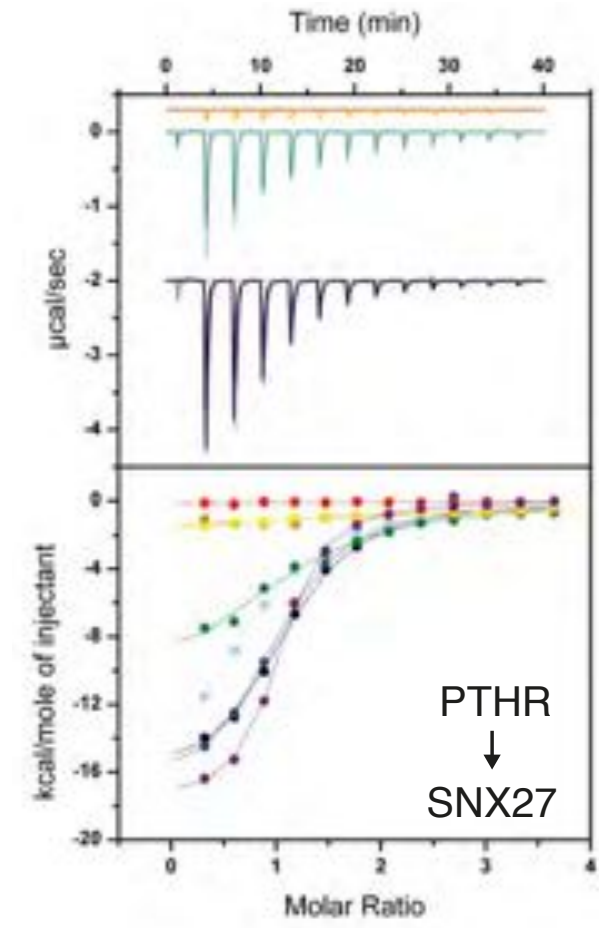

PDZbm triplet

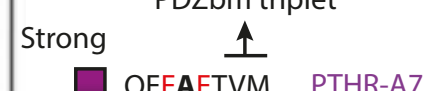

QEEWETVM PTHR-WT

QAEWETVM PTHR-A1

QEAWETVM PTHR-A2

QAAWETVM PTHR-A3

QEEWETVMA PTHR-A4*

QEEWATVM PTHR-A5*

QEAWATVM PTHR-A6

$\uparrow \uparrow$

acidic clamp

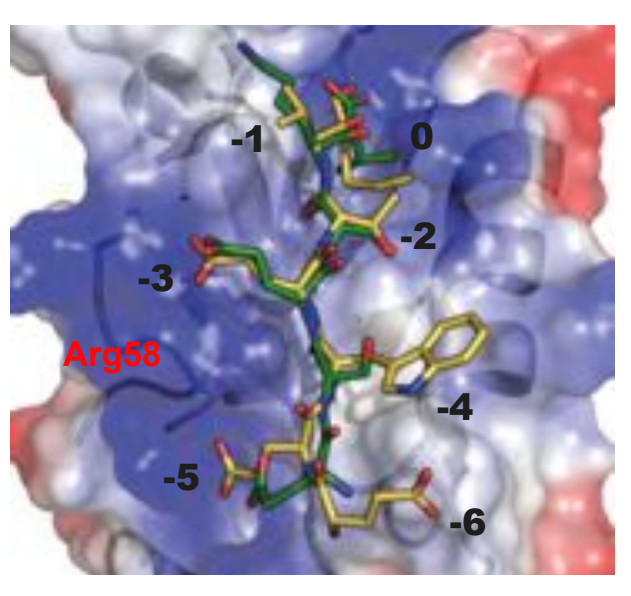

Kir3.3 PTHR

$V^{0} \quad M^{0}$

$\mathrm{K}^{-1} \mathrm{~V}^{-1}$

$\mathrm{S}^{-2} \mathrm{~T}^{-2}$

$E^{-3} E^{-3}$

$E^{-5} E^{-5}$
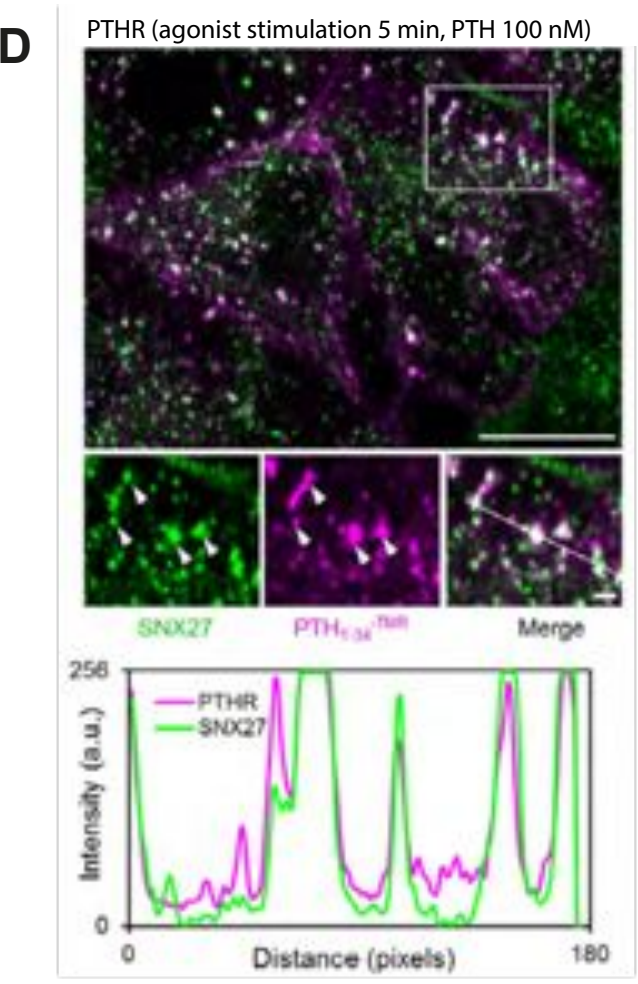

E
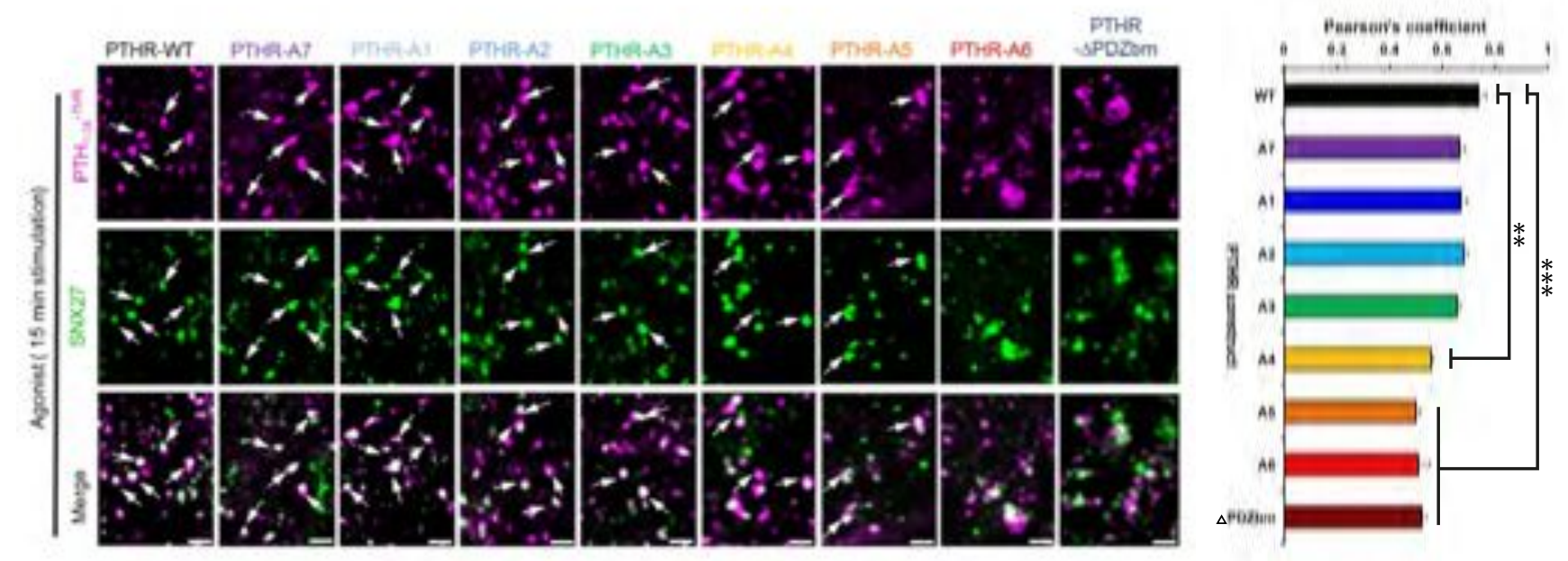

Figure 1 
A
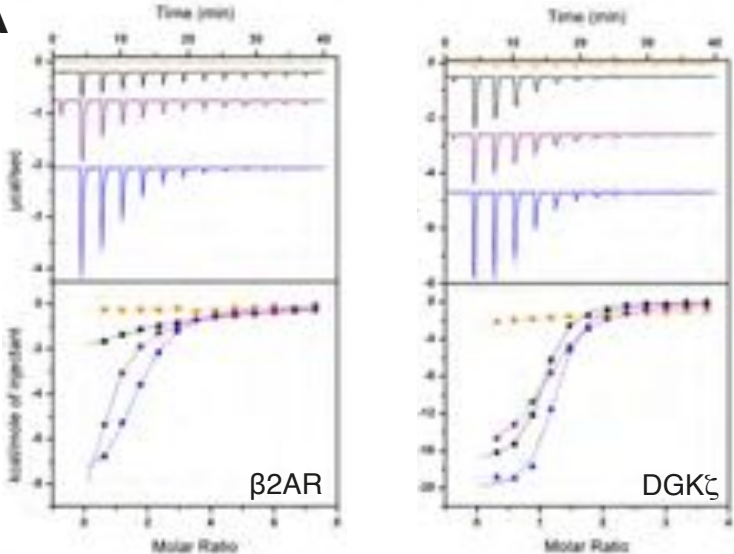

wt CSTNDSLL + SNX27PDZ

wt CSTNDSLL + SNX27PDZ

wt CSTNDSLL + SNX27PDZ/VPS26

wt REDQETAV + SNX27PDZ

Wt REDQETAV + SNX27PDZ

wt REDQETAV + SNX27PDZ/VPS26A
ss RSSQETAV + SNX27PDZ

B

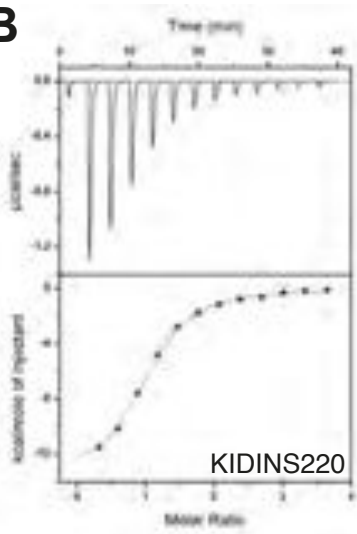

C

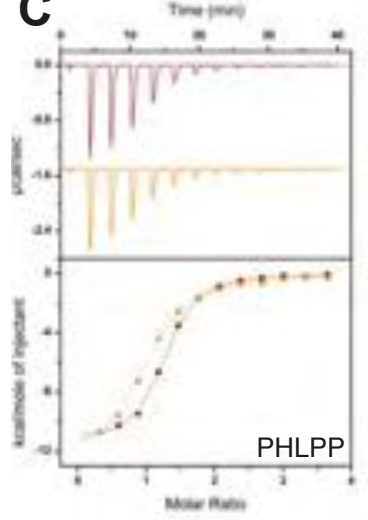

PHLPP1 PDYYDTPL + SNX27PDZ PHLPP2 PEEFDTAL + SNX27PDZ 


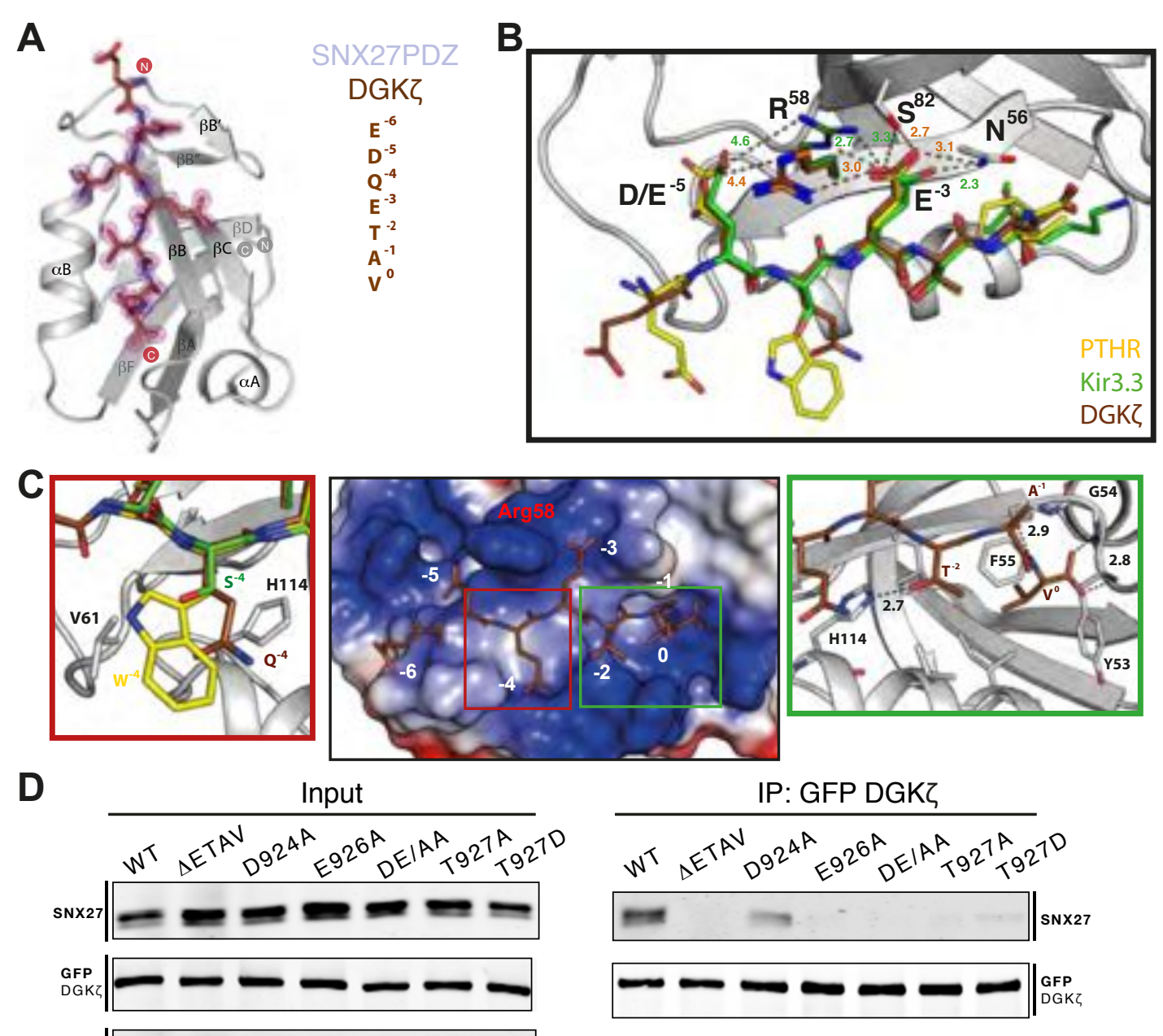

Figure 3 
A

\section{Endosomal lumen}
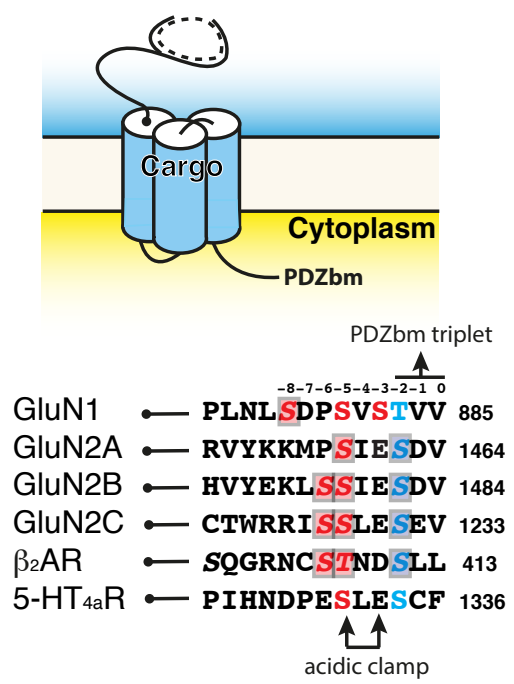

C

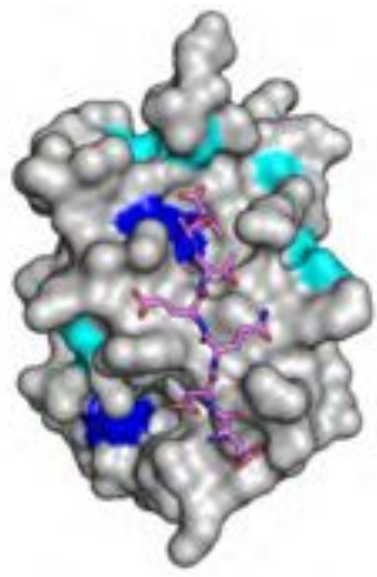

SNX27PDZ-ß2AR
B

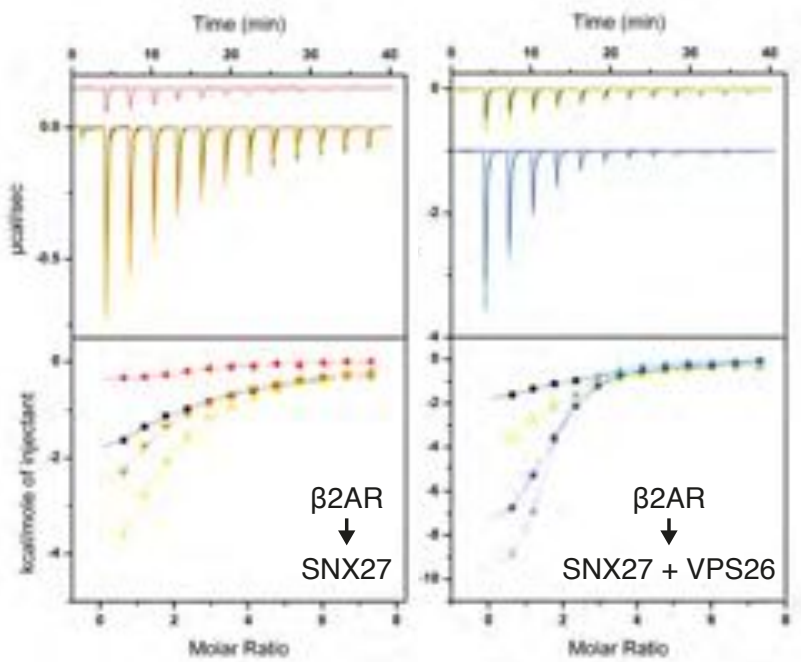

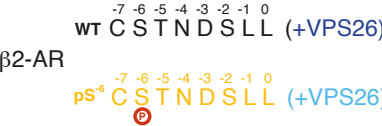

$\mathrm{PT}^{-5} \mathrm{C}^{-7} \mathrm{C}^{-6} \mathrm{C}^{-5} \mathrm{~N}^{-4} \mathrm{~N}^{-3} \mathrm{D} S \mathrm{LL}^{-1} \mathrm{~L}$

$\mathrm{pS}^{-2} \mathrm{-}^{-7} \mathrm{~S}^{-6} \mathrm{~T}^{-5} \mathrm{~N}^{-4} \mathrm{-}^{-3} \stackrel{-2}{\mathrm{~S}} \mathrm{\Theta}^{-1} L \stackrel{0}{L}$

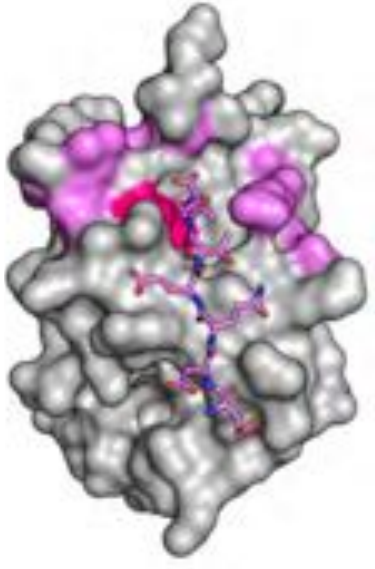

SNX27PDZ-ß2AR-pS ${ }^{-2}$

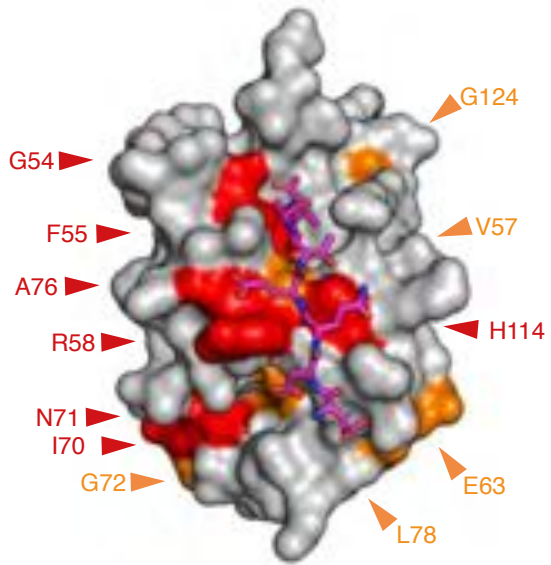

SNX27PDZ-ß2AR-pS ${ }^{-6}$ 


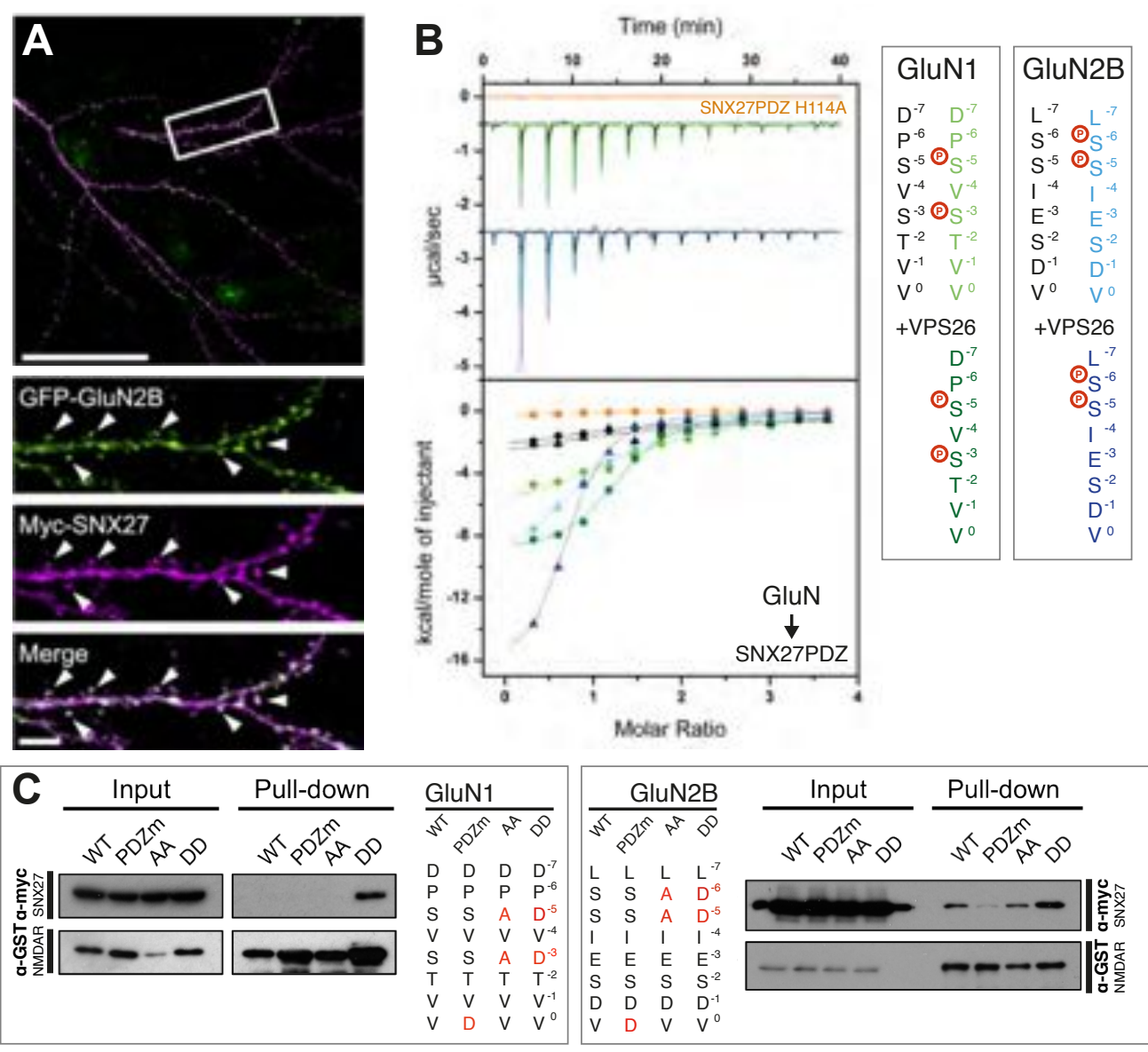

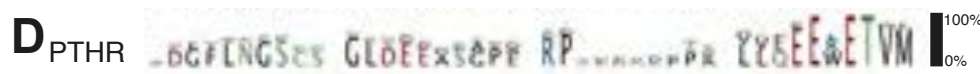

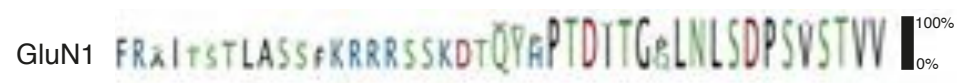

GluN2B

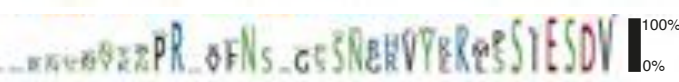


A
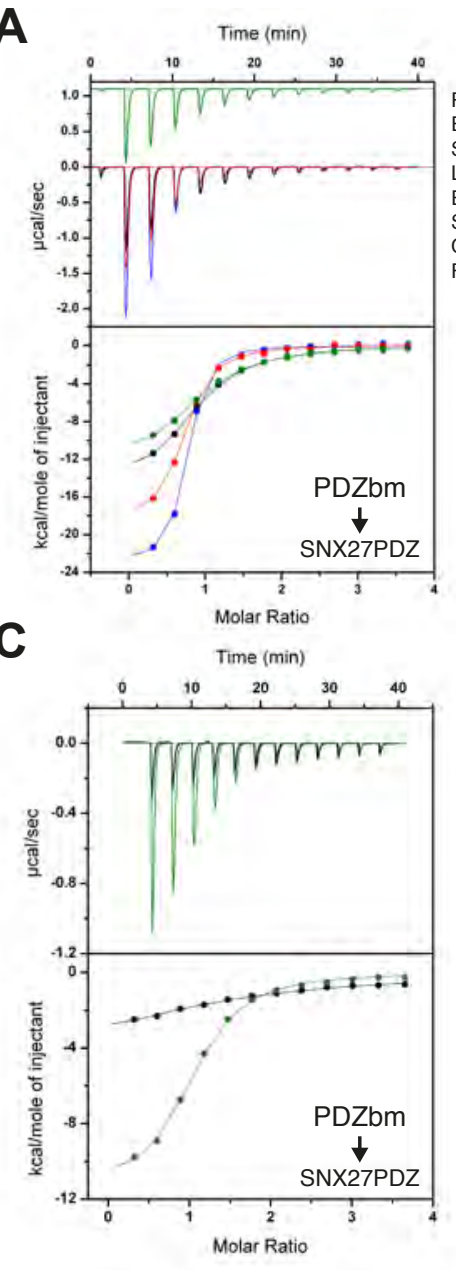

$5 \mathrm{HT}_{4(\mathrm{a})} \mathrm{R}$ $\begin{array}{lll}P^{-7} & P^{-7} & P^{-7} \\ E^{-6} & E^{-6} & E^{-6}\end{array}$ $\mathrm{E}^{-5} \overbrace{\mathrm{S}^{-5}}^{\mathrm{E}^{-6}} \mathrm{E}^{\mathrm{E}^{-5}}$ $\begin{array}{lll}E^{-3} & E^{-3} & E^{-3} \\ S^{-2} & S^{-2} & S^{-2}\end{array}$ $\mathrm{C}^{-1} \quad \mathrm{C}^{-1} \quad \mathrm{C}^{-1}$ $\mathrm{F}^{0} \mathrm{~F}^{0} \mathrm{~F}^{0}$
$+\mathrm{VPS} 26$ SNX14 $V^{-7}$
$T^{-6}$
$\mathrm{E}^{-4}$
$\mathrm{~V}^{-4}$
$\mathrm{C}^{-3}$
$\mathrm{~S}^{-2}$
$\mathrm{~W}^{-1}$
$\mathrm{M}^{0}$ LRRC3B $P^{-7}$
$D^{-6}$
$D^{-5}$
$I^{-4}$
$S^{-3}$
$T^{-2}$
$V^{-1}$
$V^{0}$
$P^{-7}$
$D^{-6}$
$D^{-5}$
$I^{-4}$
${ }^{-3}$
$T^{-2}$
$V^{-1}$
$V^{0}$

E SNX27-LRRC3B(pSer) $\mathbf{D}^{-6} \mathbf{D}^{-5} I_{\circledast}^{-4} S^{-3} T^{-2} V^{-1} V^{0}$
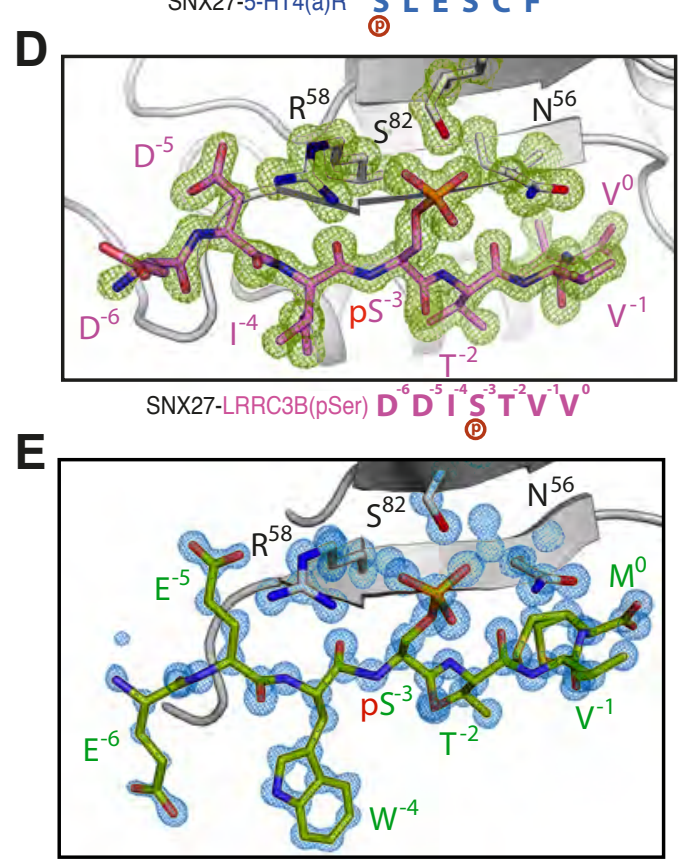

SNX27-PTHR(pSer) $E^{-6} E^{-5} \mathbf{W}^{-4} S^{-3} T^{-2} V^{-1} \mathbf{M}^{0}$

F

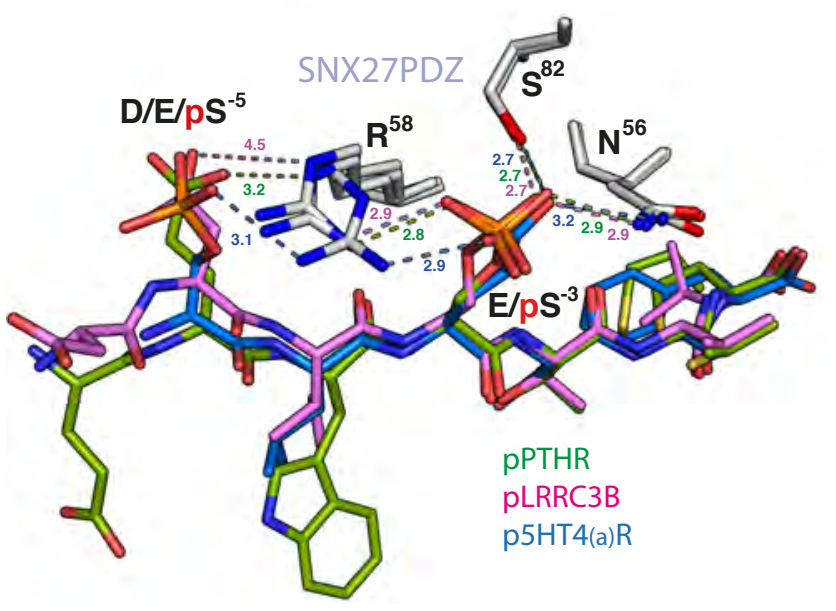

Figure 6 
A

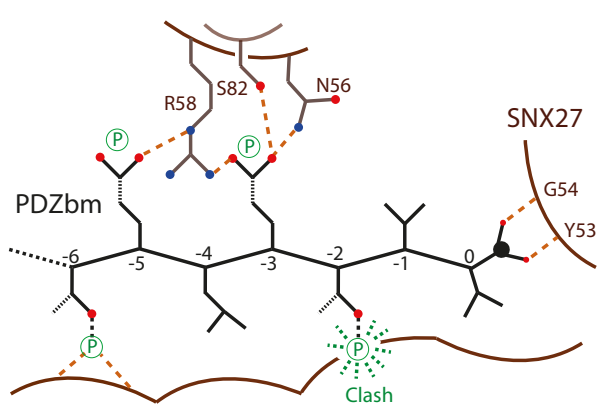

\begin{tabular}{|c|c|c|c|c|c|c|c|}
\hline & -6 & -5 & -4 & -3 & -2 & -1 & 0 \\
\hline \multirow{2}{*}{1} & $\mathbf{x}$ & $E / D$ & $\mathbf{x}$ & $E / D$ & S/T & $\mathbf{x}$ & $\Phi$ \\
\hline & ED/ST & E $E / D$ & $\mathbf{x}$ & $E / D$ & $S / T$ & $\mathbf{x}$ & $\Phi$ \\
\hline \multirow{3}{*}{2 b } & $\mathbf{x}$ & ED/ST & ${ }^{-} \mathbf{x}$ & $E / D$ & $S / T$ & $\mathbf{x}$ & $\Phi$ \\
\hline & $\mathbf{E D} / \mathbf{S T}^{\mathrm{P}}$ & ED/ST & ${ }^{P} \mathbf{x}$ & $\mathbf{S} / \mathbf{T}^{\mathrm{P}}$ & $S / T$ & $\mathbf{x}$ & $\Phi$ \\
\hline & ED/ST & $\mathbf{x}$ & $\mathbf{x}$ & $\mathbf{S} / \mathbf{T}^{\mathrm{P}}$ & $S / T$ & $\mathbf{x}$ & $\Phi$ \\
\hline \multirow{2}{*}{$3^{a}$} & $\mathbf{x}$ & $\mathbf{x}$ & $x$ & $E / D$ & $S / T$ & $\mathbf{x}$ & $\Phi$ \\
\hline & $\mathbf{x}$ & $\mathbf{x}$ & $\mathbf{x}$ & $\mathbf{S} / \mathbf{T}^{\mathrm{P}}$ & $S / T$ & $\mathbf{x}$ & $\Phi$ \\
\hline
\end{tabular}

B

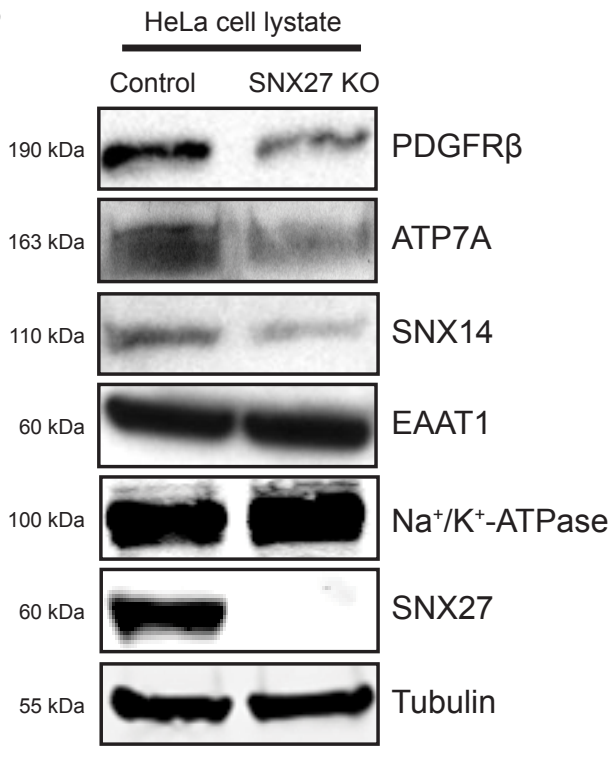

C

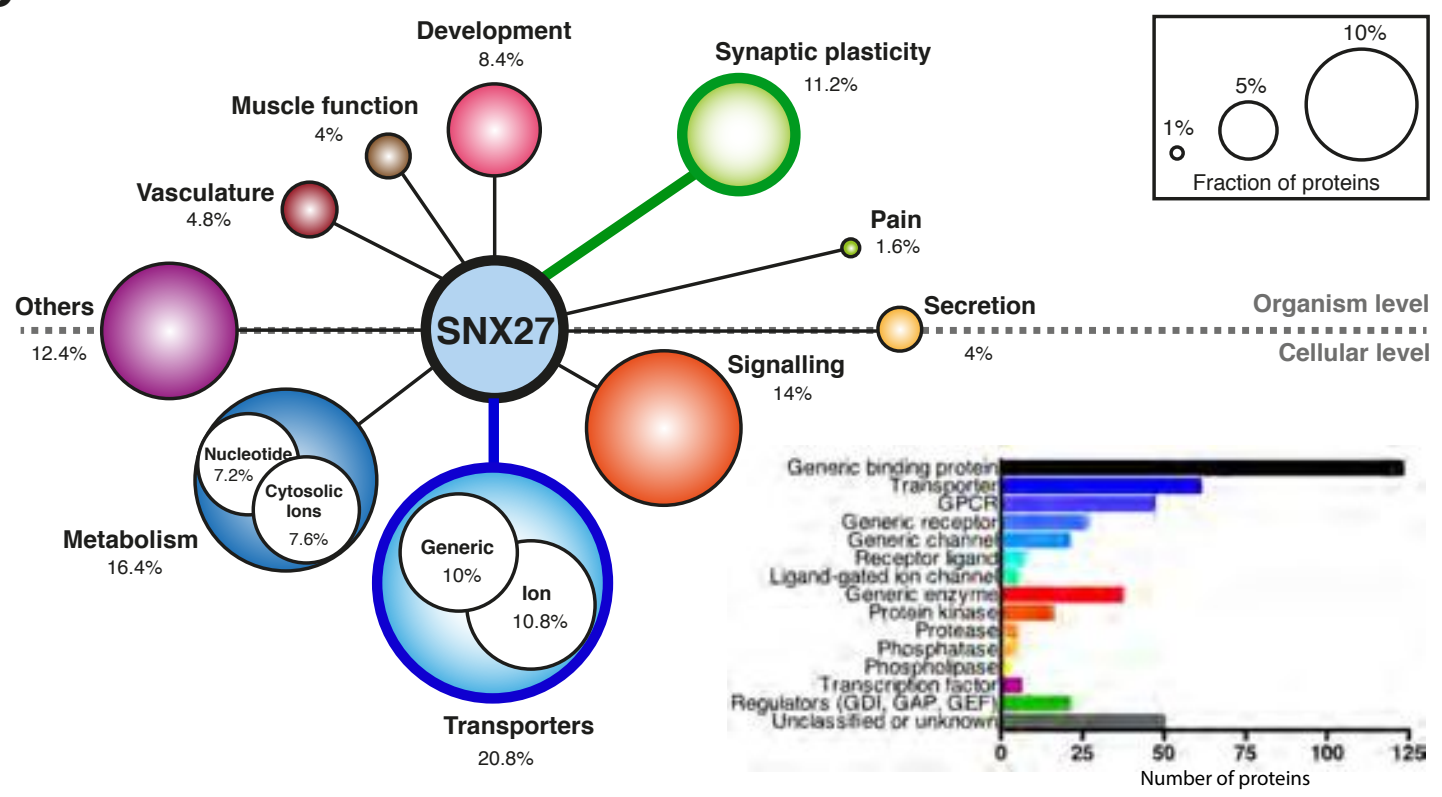


A

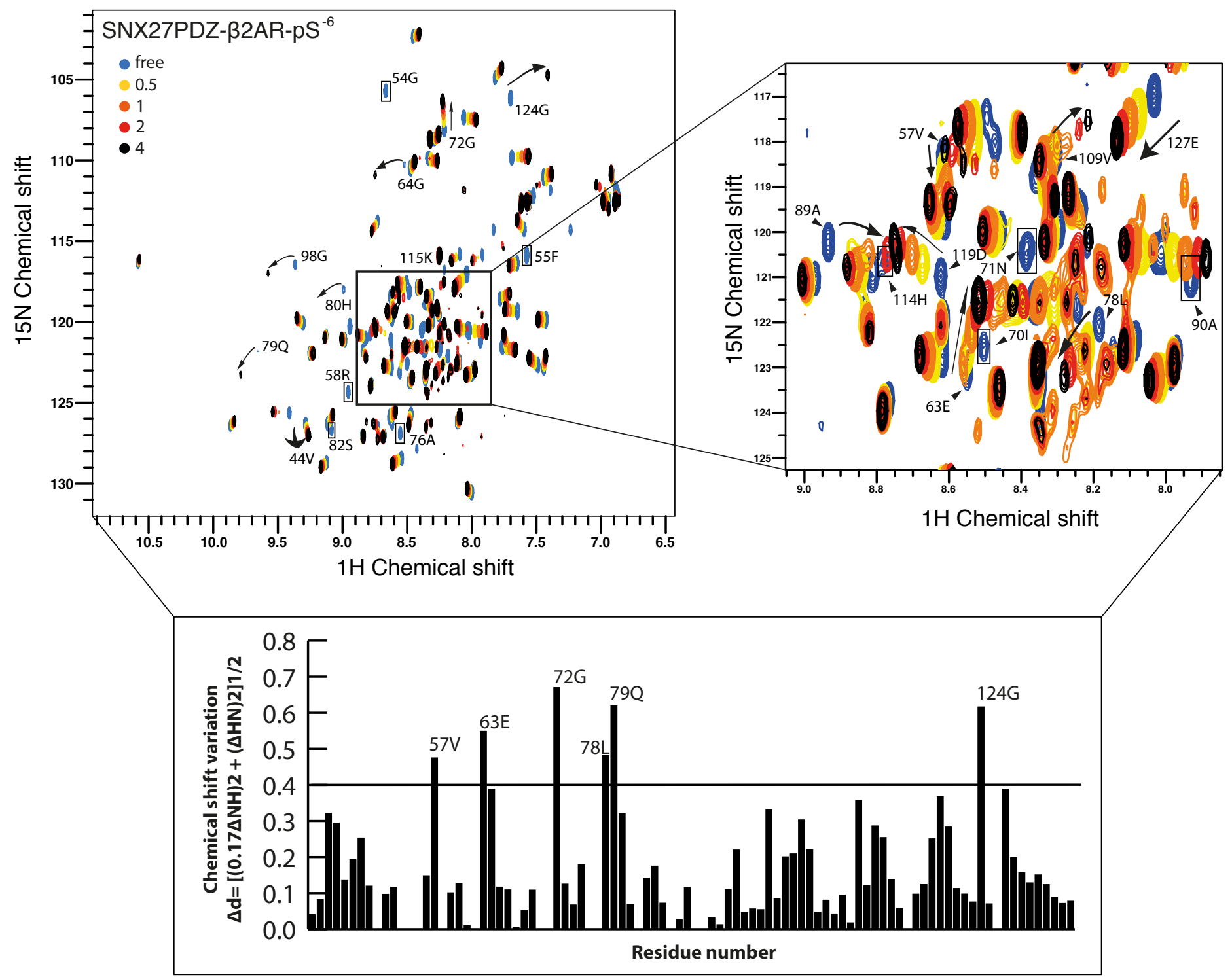

B
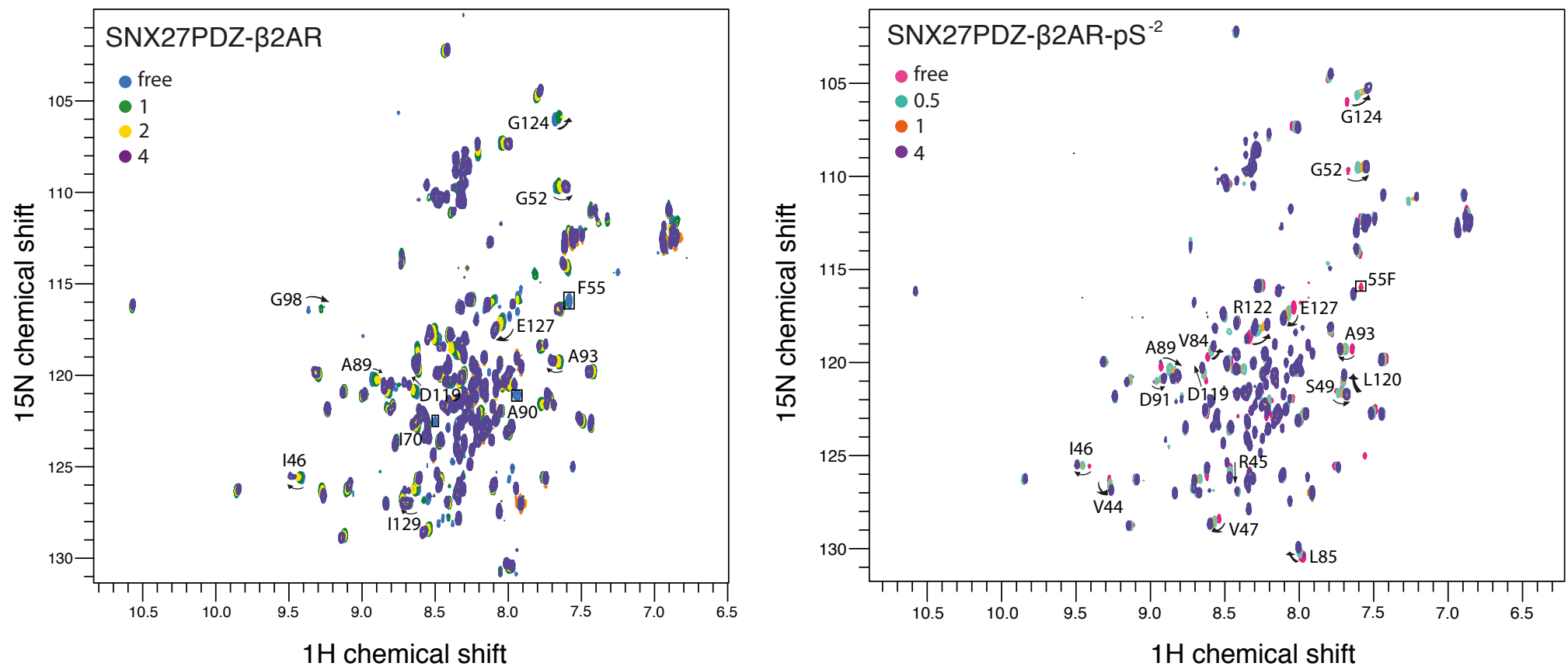

Figure S1 

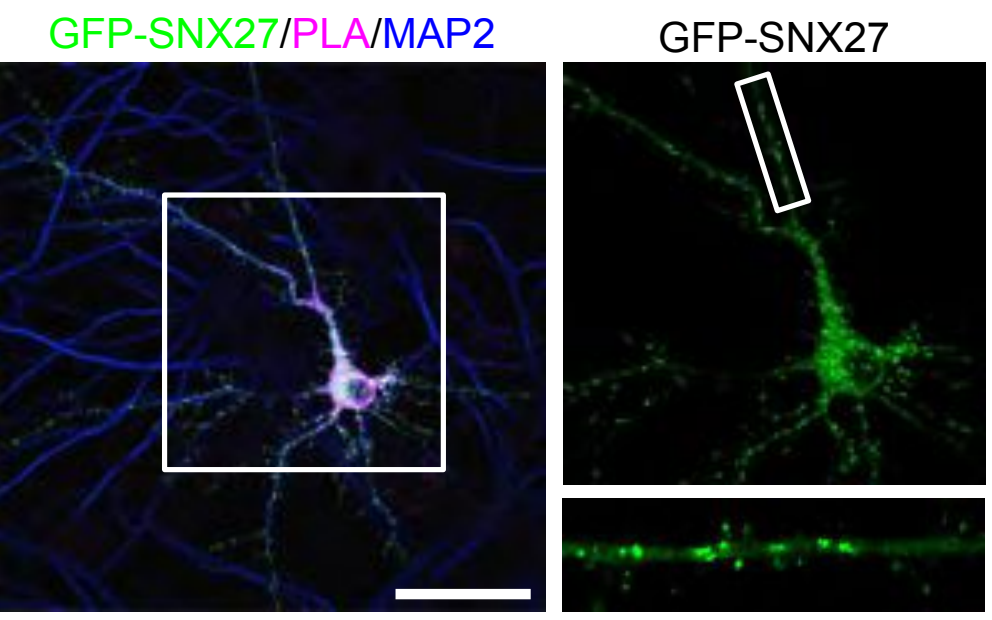

PLA

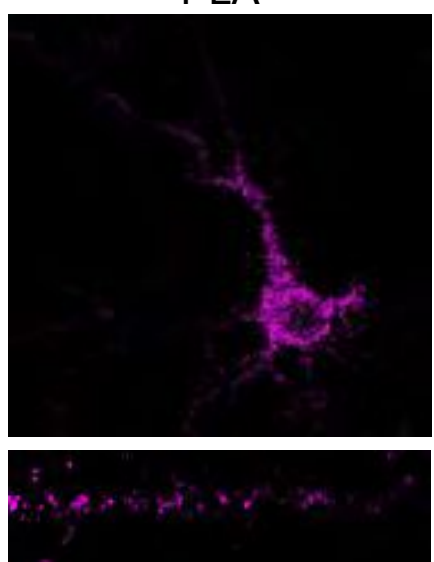

GFP-SNX27/PLA

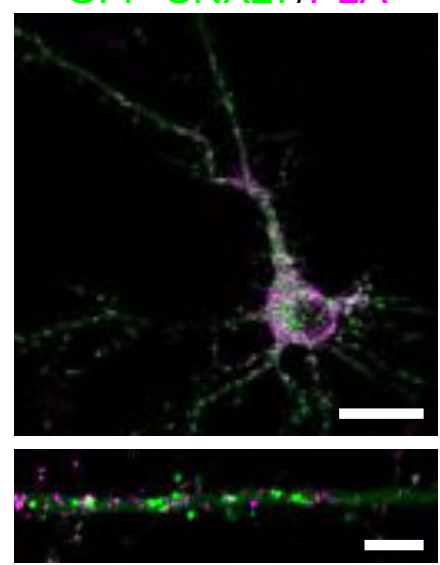

Figure S2 


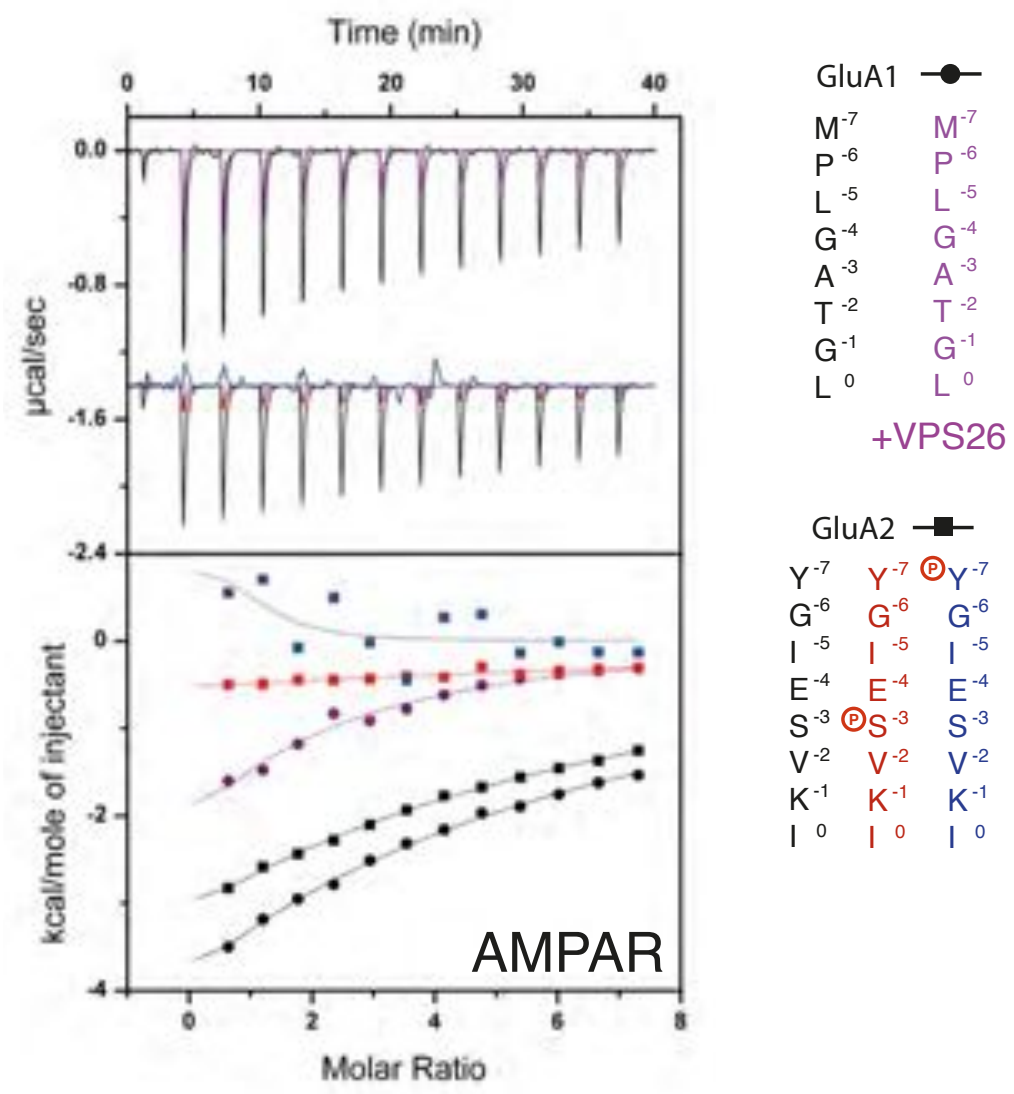

Figure S3 


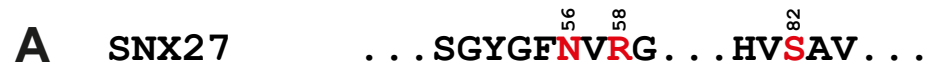

NHERF1 1 ... NGYGFHLHG. . YIRLV. . .

NHERF1_2 ...SGYGFNLHG. . . FIRSV. . .

NHERF2_1 ...QGYGFHLHG. . FIRRV . .

NHERF2 2 . Q QGYGFNLHG . . YIRSV . .

B

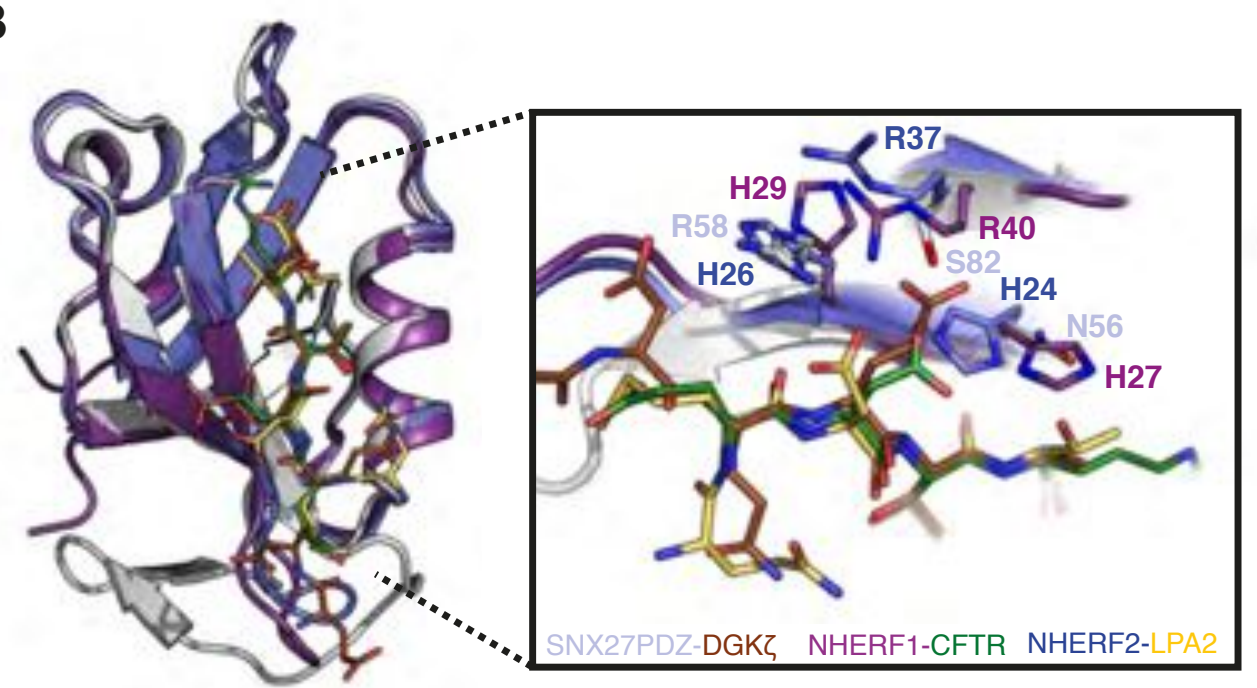

C

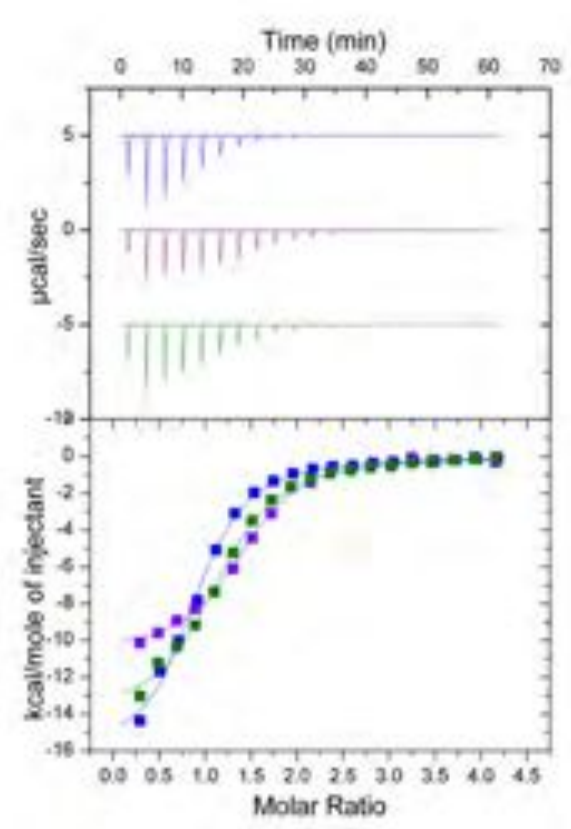

CFTR EEEVQDTRL + NHERF1 PDZ2

CFTR EEEVQDTRL + SNX27PDZ

CFTR EEEVQDTRL + SNX27PDZ/VPS26A

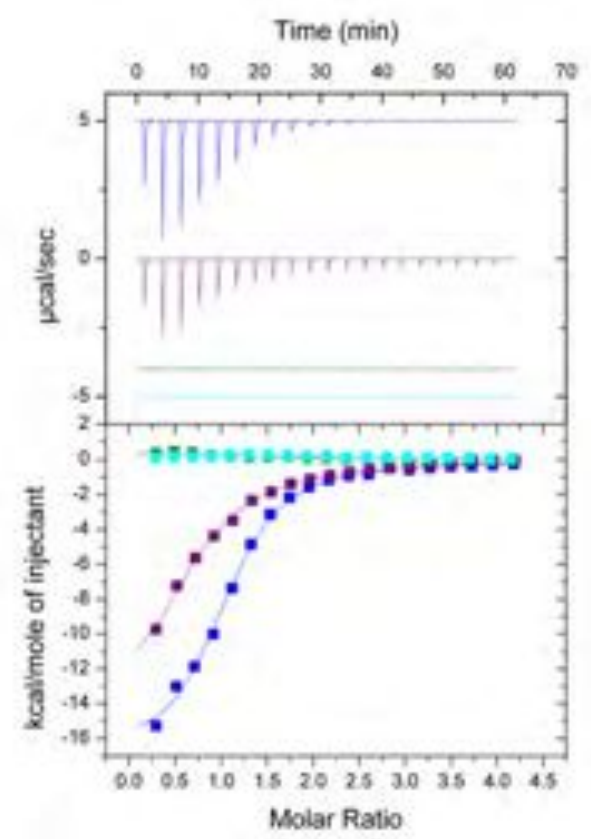

GluN2B LSSIESDV + NHERF1 PDZ2

pGluN2B LPSPSIESDV + NHERF1 PDZ2

pGluN2B LPSPSIESDV + SNX27PDZ

pGluN2B LPSPSIESDV + SNX27PDZ/VPS26A 

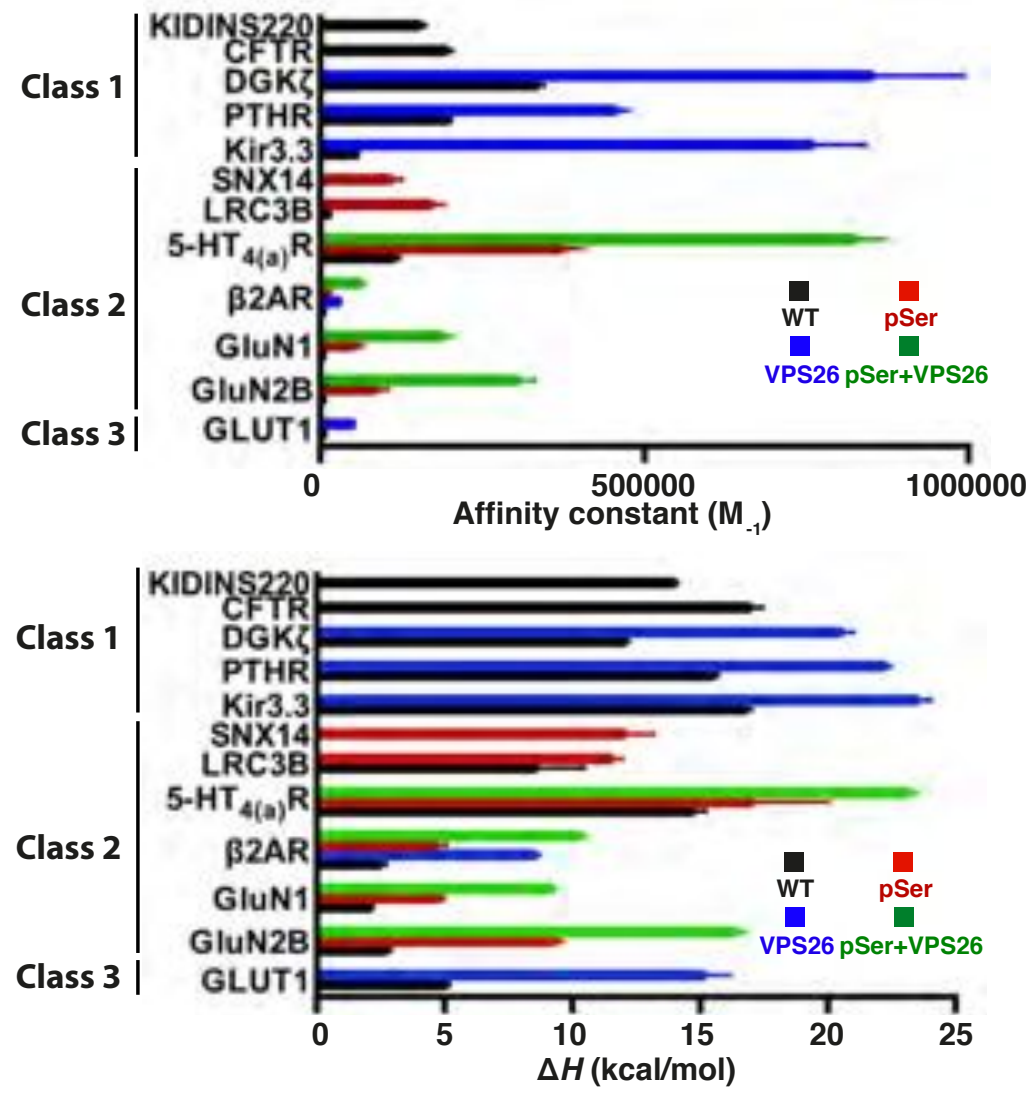

Figure S5 


\section{Black $=$ predicted}

\section{White = experimentally confirmed}

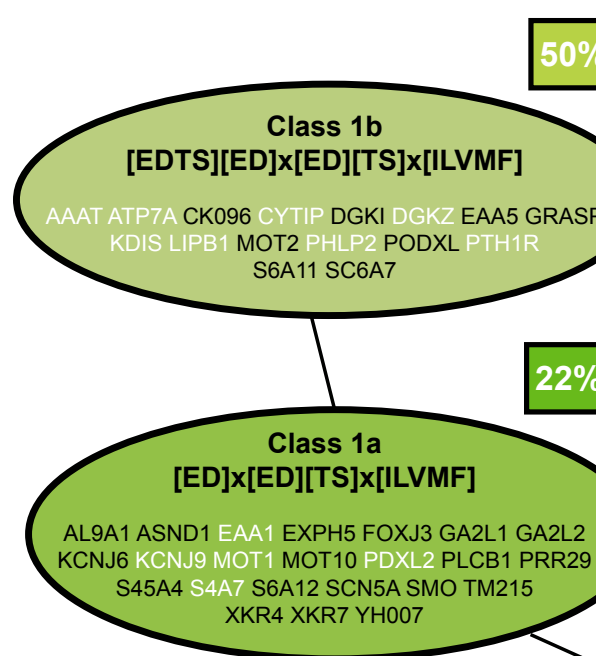

